MATHEMATICS OF COMPUTATION

229 , Pages 371-393

S 0025-5718(99)01096-0

Article electronically published on May 21, 1999

\title{
COMPUTATION OF RELATIVE CLASS NUMBERS OF CM-FIELDS BY USING HECKE $L$-FUNCTIONS
}

\author{
STÉPHANE LOUBOUTIN
}

\begin{abstract}
We develop an efficient technique for computing values at $s=1$ of Hecke $L$-functions. We apply this technique to the computation of relative class numbers of non-abelian CM-fields $\mathbf{N}$ which are abelian extensions of some totally real subfield $\mathbf{L}$. We note that the smaller the degree of $\mathbf{L}$ the more efficient our technique is. In particular, our technique is very efficient whenever instead of simply choosing $\mathbf{L}=\mathbf{N}^{+}$(the maximal totally real subfield of $\mathbf{N}$ ) we can choose $\mathbf{L}$ real quadratic. We finally give examples of computations of relative class numbers of several dihedral CM-fields of large degrees and of several quaternion octic CM-fields with large discriminants.
\end{abstract}

\section{INTRODUCTION}

In [Lou6] we developed a general technique for computing relative class numbers $h_{\mathbf{N}}^{-}$of CM-fields $\mathbf{N}$ based on the use of (8) and Theorem 2 below with $\mathbf{L}=\mathbf{N}^{+}$and $\chi=\chi_{\mathbf{N} / \mathbf{N}^{+}}$, in which case one can easily prove that $W_{\chi}=1$ (use the functional equations satisfied by $\zeta_{\mathbf{N}}$ and $\zeta_{\mathbf{N}^{+}}$). This technique has been used to compute relative class numbers of various non-abelian CM-fields of degree 4, 6, 8, 12 or 16 (see [Lou2], [Lou3], [Lou4], [LO] and [LOO]), and these computations were in turn used to settle the class number one problem for the non-abelian normal CM-fields of degree $\leq 16$. However, this technique becomes too slow when the degree and discriminant of $\mathbf{N}$ become larger. Indeed, setting

$$
B(\mathbf{N})=A_{\mathbf{N} / \mathbf{N}^{+}}\left(\frac{\lambda}{n} \log A_{\mathbf{N} / \mathbf{N}^{+}}\right)^{n / 2}, \quad \text { where } A_{\mathbf{N} / \mathbf{N}^{+}}=\sqrt{d_{\mathbf{N}} / \pi^{n} d_{\mathbf{N}^{+}}},
$$

we proved that if $\lambda>1$ and $n$ are fixed, then, according to (8) and (11), we should compute $B(\mathbf{N})$ terms in the absolutely convergent series (10) (with $\chi=\chi_{\mathbf{N} / \mathbf{N}^{+}}$) to compute $h_{\mathbf{N}}^{-}$when $\mathbf{N}$ ranges over a familly of CM-fields of degree $2 n$. In particular, this technique is too slow to compute relative class numbers of dihedral CM-fields of degree $4 p$ when the prime $p$ is not that large, say equal to 5 or 7 .

The main prospect of this paper is to explain how (8) and Theorem 2 below make it easy to compute the relative class numbers of such dihedral CM-fields $\mathbf{N}$ by computing the values at $s=1$ of $(p-1) / 2$ Hecke $L$-functions over the real quadratic subfield $\mathbf{L}$ of $\mathbf{N}$. According to Theorems 4 and 7 , to get a real

Received by the editor April 16, 1997.

1991 Mathematics Subject Classification. Primary 11M20, 11R42; Secondary 11R29.

Key words and phrases. CM-field, relative class number, Hecke $L$-function, ray class field, dihedral field. 
approximation of the integer $h_{\mathbf{N}}^{-}$close enough to it to enable us to deduce its exact value, we have to compute much fewer than $B(\mathbf{N})$ terms in each of the $(p-1) / 2$ absolutely convergent series (given in Theorem 4) whose limits are the values at $s=1$ of these Hecke $L$-functions (see also our third example). Subsections 4.1, 4.2 and 4.3 will provide the reader with actual computations of Hecke $L$-functions over real quadratic fields. Note that according to Theorem 2, there is nothing peculiar to $\mathbf{L}$ being a real quadratic field. It is just that it is easier to compute in ray class fields of quadratic fields than to compute in ray class fields of number fields of higher degree. In fact, in using Theorem 2 below and [Lou6] to get a practical technique for computing values of $K_{m, 1}$ and $K_{m, 2}$ (i.e., to get a generalization of Theorems 6 and 17), it would be easy to generalize all our results to cases where $\mathbf{L}$ is not quadratic (in the last section of this paper we work out an example of calculation of a Hecke $L$-function over a real cubic field). Hence, it is worth noticing that our technique is much more general than the one developed in [Mey], which assumes L quadratic. Our technique is also more efficient than the one developed in [Oka1] and [Oka2] based on the results in [Shi]. Indeed, a close look at [Oka1] shows that if the fundamental unit

$$
\epsilon=\left(x_{\epsilon}+y_{\epsilon} \sqrt{d_{\mathbf{L}}}\right) / 2
$$

of a real quadratic field $\mathbf{L}$ is large, which happens quite often (for example, if $d_{\mathbf{L}}=18361$ then $y_{\epsilon}$ has 49 digits in base 10), then Shintani's method becomes too slow to be of any practical use for numerical computations, for it requires a number of operations growing to infinity at least linearly in the size of $y_{\epsilon}$ to compute values of Hecke $L$-functions over $\mathbf{L}$ at $s=1$. Even in the case where $y_{\epsilon}$ is small, Shintani's method is less efficient than the one developed here (see subsection 4.3).

To conclude, the technique here developed is more efficient but less simple than the one used in [Lou4] and [Lou6]. Indeed, if $\mathbf{N}$ is a normal CM-field, we only have to know how to compute the inertia and residual degrees in $\mathbf{N}$ of any prime $p$ to be able to use the method developed in [Lou6]. Here, we need a precise description of the Hecke characters associated to the abelian extension $\mathbf{N} / \mathbf{L}$, and we must determine the values of all the $W_{\chi}$ 's associated to these Hecke characters. However, the present method is much more efficient.

\section{Numerical computation of $L(1, \chi)$}

Let $\mathcal{C}=\infty_{1} \infty_{2} \cdots \infty_{t} \mathcal{C}_{0}$ be a cycle of a number field $\mathbf{L}$, where $\mathcal{C}_{0}$, the finite part of $\mathcal{C}$, is an integral ideal of $\mathbf{L}$ and where $\infty_{i}, 1 \leq i \leq t$, are $t$ distinct real places of $\mathbf{L}$. For simplicity of notation, $N_{\mathbf{L} / \mathbf{Q}}(\mathcal{C})$ and $N_{\mathbf{L} / \mathbf{Q}}\left(\mathcal{C}_{0}\right)$ will both denote the absolute norm of the ideal $\mathcal{C}_{0}$. Let $\mathrm{CL}_{\mathbf{L}}(\mathcal{C})$ denote the group of $\mathcal{C}$-ideal classes, which is also called the unit ray class group of $\mathbf{L}$ modulo $\mathcal{C}$. If $\mathcal{C}^{\prime}$ divides $\mathcal{C}$, then the canonical map $\mathrm{CL}_{\mathbf{L}}(\mathcal{C}) \longrightarrow \mathrm{CL}_{\mathbf{L}}\left(\mathcal{C}^{\prime}\right)$ is onto and any character $\chi^{\prime}$ on $\mathrm{CL}_{\mathbf{L}}\left(\mathcal{C}^{\prime}\right)$, i.e., any morphism of multiplicative groups $\chi^{\prime}: \mathrm{CL}_{\mathbf{L}}\left(\mathcal{C}^{\prime}\right) \longrightarrow \mathbf{C}^{*}$, induces a character $\chi$ on $\mathrm{CL}_{\mathbf{L}}(\mathcal{C})$. We say that a character $\chi$ on $\mathrm{CL}_{\mathbf{L}}(\mathcal{C})$ is primitive if it is not induced by any character $\chi^{\prime}$ on $\mathrm{CL}_{\mathbf{L}}\left(\mathcal{C}^{\prime}\right)$ for any $\mathcal{C}^{\prime} \neq \mathcal{C}$ dividing $\mathcal{C}$. In that situation, $\mathcal{C}$ is called the conductor of $\chi$ and we write $\mathcal{C}=\mathcal{F}_{\chi}$.

Let $\mathbf{E} / \mathbf{L}$ be an abelian extension of number fields of conductor $\mathcal{C}=\mathcal{F}_{\mathbf{E} / \mathbf{L}}($ a cycle of $\mathbf{L}$ ). According to class field theory, $\mathbf{E}$ is a subfield of the unit ray class field $\mathbf{R}_{\mathbf{L}}(\mathcal{C})$ of $\mathbf{L}$ where from the Artin map we get an isomorphism $\operatorname{Gal}\left(\mathbf{R}_{\mathbf{L}}(\mathcal{C}) / \mathbf{L}\right) \simeq \mathrm{CL}_{\mathbf{L}}(\mathcal{C})$. We let $X_{\mathbf{E} / \mathbf{L}}$ denote the group of primitive characters of conductors dividing $\mathcal{C}=$ $\mathcal{F}_{\mathbf{E} / \mathbf{L}}$ which induce the group of (not necessarily primitive) characters on $\mathrm{CL}_{\mathbf{L}}(\mathcal{C})$ 
which are trivial on $\operatorname{Gal}\left(\mathbf{R}_{\mathbf{L}}(\mathcal{C}) / \mathbf{N}\right)$. We have the following factorization of the Dedekind zeta function of $\mathbf{E}$ :

$$
\zeta_{\mathbf{E}}(s)=\prod_{\chi \in X_{\mathbf{E} / \mathbf{L}}} L(s, \chi) .
$$

Let $\mathbf{N}$ be a number field. We say that $\mathbf{N}$ is a CM-field if $\mathbf{N}$ is totally imaginary and if $\mathbf{N}$ is a quadratic extension of its maximal totally real subfield $\mathbf{N}^{+}$. In that situation, the degree $2 n$ of $\mathbf{N}$ is even and the class number $h_{\mathbf{N}^{+}}$of $\mathbf{N}^{+}$divides the class number $h_{\mathbf{N}}$ of $\mathbf{N}$, and the ratio $h_{\mathbf{N}}^{-}=h_{\mathbf{N}} / h_{\mathbf{N}^{+}}$is called the relative class number of $\mathbf{N}$. Let $Q_{\mathbf{N}} \in\{1,2\}, w_{\mathbf{N}}, d_{\mathbf{N}}$ and $d_{\mathbf{N}^{+}}$denote the Hasse unit index of $\mathbf{N}$, the number of roots of unity in $\mathbf{N}$ and the absolute values of the discriminants of $\mathbf{N}$ and $\mathbf{N}^{+}$, respectively. We have (see [Wa])

$$
h_{\mathbf{N}}^{-}=\frac{Q_{\mathbf{N}} w_{\mathbf{N}}}{(2 \pi)^{n}} \sqrt{\frac{d_{\mathbf{N}}}{d_{\mathbf{N}^{+}}}} \frac{\operatorname{Res}_{s=1}\left(\zeta_{\mathbf{N}}\right)}{\operatorname{Res}_{s=1}\left(\zeta_{\mathbf{N}^{+}}\right)} .
$$

From now on, we assume that $\mathbf{N}$ is a CM-field of degree $2 n$ and that $\mathbf{N}$ is an abelian extension of some of its totally real subfields $\mathbf{L}$ of degree $m$. Let $\mathcal{C}=$ $\mathcal{F}_{\mathbf{N} / \mathbf{L}}=\infty_{1} \cdots \infty_{m} \mathcal{C}_{\mathbf{N} / \mathbf{L}}$ denote the conductor of this abelian extension $\mathbf{N} / \mathbf{L}$, and let $\mathcal{C}^{+}=\mathcal{F}_{\mathbf{N}^{+} / \mathbf{L}}$ denote the conductor of the abelian subextension $\mathbf{N}^{+} / \mathbf{L}$. Therefore, $\mathcal{C}^{+}$divides $\mathcal{C}, \mathbf{R}_{\mathbf{L}}\left(\mathcal{C}^{+}\right)$is a subfield of $\mathbf{R}_{\mathbf{L}}(\mathcal{C})$ and $X_{\mathbf{N}^{+} / \mathbf{L}}$ is a subgroup of the group $X_{\mathbf{N} / \mathbf{L}}$. We set $X_{\mathbf{N} / \mathbf{L}}^{-}=X_{\mathbf{N} / \mathbf{L}} \backslash X_{\mathbf{N}^{+} / \mathbf{L}}$. Notice that the conductor $\mathcal{F}_{\chi}$ of any $\chi$ in $X_{\mathbf{N} / \mathbf{L}}^{-}$is of the form $\mathcal{F}_{\chi}=\infty_{1} \cdots \infty_{m} \mathcal{C}_{\chi}$ for some $\mathcal{C}_{\chi}$ dividing $\mathcal{C}_{\mathbf{N} / \mathbf{L}}$. Using (2) for both $\mathbf{N}$ and $\mathbf{N}^{+}$, we get

$$
\left(\zeta_{\mathbf{N}} / \zeta_{\mathbf{N}^{+}}\right)(s)=\prod_{\chi \in X_{\mathbf{N} / \mathbf{L}}^{-}} L(s, \chi)
$$

and

$$
h_{\mathbf{N}}^{-}=\frac{Q_{\mathbf{N}} w_{\mathbf{N}}}{(2 \pi)^{n}} \sqrt{\frac{d_{\mathbf{N}}}{d_{\mathbf{N}^{+}}}} \prod_{\chi \in X_{\mathbf{N} / \mathbf{L}}^{-}} L(1, \chi) .
$$

Note that there are $[\mathbf{N}: \mathbf{L}] / 2=n / m$ characters in $X_{\mathbf{N} / \mathbf{L}}^{-}$. It is known that if $\chi$ is a primitive character of conductor $\mathcal{C}=\infty_{1} \cdots \infty_{m} \mathcal{C}_{\chi}$ which is ramified at all the $m$ infinite places of $\mathbf{L}$, then the Hecke $L$-function $s \mapsto L(s, \chi)$ satisfies the functional equation

$$
F(1-s, \chi)=W_{\chi} F(s, \bar{\chi})
$$

for some Artin root number $W_{\chi}$ with $\left|W_{\chi}\right|=1$, where

$$
F(s, \chi)=A_{\chi}^{s} \Gamma^{m}((s+1) / 2) L(s, \chi)
$$

and

$$
A_{\chi}=\sqrt{d_{\mathbf{L}} N_{\mathbf{L} / \mathbf{Q}}\left(\mathcal{C}_{\chi}\right) / \pi^{m}}
$$

Let $\chi_{\mathbf{N} / \mathbf{N}^{+}}$denote the quadratic Hecke character of conductor $\mathcal{F}_{\mathbf{N} / \mathbf{N}^{+}}$associated to the quadratic extension $\mathbf{N} / \mathbf{N}^{+}$. We have

$$
A_{\chi_{\mathbf{N} / \mathbf{N}^{+}}}=\sqrt{d_{\mathbf{N}^{+}} N_{\mathbf{N}^{+} / \mathbf{Q}}\left(\mathcal{F}_{\mathbf{N} / \mathbf{N}^{+}}\right) / \pi^{n}}=\sqrt{d_{\mathbf{N}} / \pi^{n} d_{\mathbf{N}^{+}}}=\prod_{\chi \in X_{\mathbf{N} / \mathbf{L}}^{-}} A_{\chi},
$$


and we obtain

$$
h_{\mathbf{N}}^{-}=2^{-n} \pi^{-n / 2} Q_{\mathbf{N}} w_{\mathbf{N}} \prod_{\chi \in X_{\mathbf{N} / \mathbf{L}}^{-}} A_{\chi} L(1, \chi) .
$$

For $\Re(s)>1$, write

$$
L(s, \chi)=\sum_{n \geq 1} \frac{a_{n}(\chi)}{n^{s}}
$$

where

$$
a_{n}(\chi)=\sum_{N_{\mathbf{L} / \mathbf{Q}}(\mathbf{I})=n} \chi(\mathbf{I})
$$

(this sum ranges over all the nonzero integral ideals of $\mathbf{L}$ of norm $n$, and we set $\chi(\mathbf{I})=0$ if $\mathbf{I}$ is not coprime with $\left.\mathcal{F}_{\chi}\right)$. According to $(9), n \mapsto a_{n}(\chi)$ is multiplicative, i.e., $\operatorname{gcd}(m, n)=1$ implies $a_{m n}(\chi)=a_{m}(\chi) a_{n}(\chi)$. Therefore, when doing actual computation we will only explain how we compute $a_{p^{k}}(\chi)$ on powers of primes. Using (5) and the Euler product of $s \mapsto L(s, \chi)$, the reader can easily prove

Lemma 1. If all the $a_{p^{k}}(\chi)$ are real, then all the $a_{n}(\chi)$ are real, $W_{\chi}$ is equal to +1 or $-1, L(1, \chi)$ is real, and $L(1, \chi)>0$.

Theorem 2. Let $\mathbf{L}$ be a totally real number field of degree $m$ and let $\chi$ be a primitive Hecke character of conductor $\mathcal{C}=\infty_{1} \infty_{2} \cdots \infty_{m} \mathcal{C}_{\chi}$ on the ray class group $\mathrm{CL}_{\mathbf{L}}(\mathcal{C})$ for some integral ideal $\mathcal{C}_{\chi}$ of $\mathbf{L}$. In particular, we assume that $\chi$ is ramified at all the infinite places of $\mathbf{L}$. Let $W_{\chi}$ be the Artin root number which appears in the functional equation of the Hecke L-function $s \mapsto L(s, \chi)$. For $\alpha>1$ set

$$
K_{m, 1}(B)=\frac{1}{2 \pi i} \int_{\alpha-i \infty}^{\alpha+i \infty} \Gamma^{m}(s) \frac{B^{2-2 s}}{s-1} d s
$$

and

$$
K_{m, 2}(B)=\frac{1}{2 \pi i} \int_{\alpha-i \infty}^{\alpha+i \infty} \Gamma^{m}(s) \frac{B^{2-2 s}}{s-(1 / 2)} d s .
$$

Then

$$
L(1, \chi)=\sum_{n \geq 1} \frac{a_{n}(\chi)}{n} K_{m, 1}\left(n / A_{\chi}\right)+W_{\chi} \sum_{n \geq 1} \frac{\overline{a_{n}(\chi)}}{n} K_{m, 2}\left(n / A_{\chi}\right),
$$

where $A_{\chi}=\sqrt{d_{L} N_{L / Q}\left(\mathcal{C}_{\chi}\right) / \pi^{m}}$, and since for $B>0$ we have

$$
0 \leq K_{m, 2}(B) \leq K_{m, 1}(B) \leq m e^{-B^{2 / m}},
$$

both the series which appear in (10) are absolutely convergent.

Proof. Set

$$
\left.H_{m}(x)=\frac{1}{2 \pi i} \int_{\alpha-i \infty}^{\alpha+i \infty} \Gamma^{m}((s+1) / 2)\right) x^{-s} d s \quad(x>0 \quad \text { and } \alpha>0)
$$

and

$G(x, \chi)=\frac{1}{2 \pi i} \int_{\alpha-i \infty}^{\alpha+i \infty} F(s, \chi) x^{-s} d s=\sum_{n \geq 1} a_{n}(\chi) H_{m}\left(n x / A_{\chi}\right) \quad(x>0$ and $\alpha>1)$. 
Using the same line of reasoning as in [Lou4] and [Lou6], we obtain

$$
\begin{gathered}
G(x, \chi)=\frac{W_{\chi}}{x} G(1 / x, \bar{\chi}) \quad(x>0) \\
F(s, \chi)=\int_{0}^{\infty} G(x, \chi) x^{s} \frac{d x}{x}=\int_{1}^{\infty} G(x, \chi) x^{s-1} d x+W_{\chi} \int_{1}^{\infty} G(x, \bar{\chi}) x^{-s} d x,
\end{gathered}
$$

and

$$
\begin{aligned}
& A_{\chi} L(1, \chi)=F(1, \chi) \\
& \quad=\sum_{n \geq 1} a_{n}(\chi) \int_{1}^{\infty} H_{m}\left(n x / A_{\chi}\right) d x+W_{\chi} \sum_{n \geq 1} \overline{a_{n}(\chi)} \int_{1}^{\infty} H_{m}\left(n x / A_{\chi}\right) \frac{d x}{x} .
\end{aligned}
$$

Noticing that

$$
K_{m, 1}(B)=\int_{B}^{\infty} H_{m}(x) d x \quad \text { and } \quad K_{m, 2}(B)=B \int_{B}^{\infty} H_{m}(x) \frac{d x}{x},
$$

we get (10).

As for the proof of (11), we refer the reader to [Lou6, 3. Proof of Proposition 1]. Notice that $K_{m, 1}(B)+K_{m, 2}(B)=K_{m}(B)$, where $m \mapsto K_{m}(B)$ is the function used in [Lou6].

\section{HeCKe $L$-FUnCTIONS OVER REAL QUADRATIC FIELDS}

Let $\mathbf{L}$ be a real quadratic number field. Let $\mathbf{A}_{\mathbf{L}}, d_{\mathbf{L}}, h_{\mathbf{L}}, \chi_{\mathbf{L}}$ and $\infty_{1}$ and $\infty_{2}$ denote its ring of algebraic integers, the absolute value of its discriminant, its class number, its primitive modulo $d_{\mathbf{L}}$ quadratic character and the two infinite places of L. Let $\mathcal{F}_{0}$ be an integral ideal of $\mathbf{L}$, set $\mathcal{F}=\infty_{1} \infty_{2} \mathcal{F}_{0}$, and let $\chi$ be a primitive Hecke character on $\mathrm{Cl}_{\mathbf{L}}(\mathcal{F})$, the ray class group modulo $\mathcal{F}$. In particular, we assume that $\chi$ is ramified at both $\infty_{1}$ and $\infty_{2}$. It is known that if we restrict $\chi$ to principal ideals $(\alpha)$ coprime with $\mathcal{F}_{0}$, then there exists a unique character $\chi_{0}$ on $\left(\mathbf{A}_{\mathbf{L}} / \mathcal{F}_{0}\right)^{*}$ such that

$$
\chi((\alpha))=\nu(\alpha) \chi_{0}(\alpha)
$$

where for any $\alpha \in \mathbf{L} \backslash\{0\}$ we let $\nu(\alpha)$ denote the sign of $N_{\mathbf{L} / \mathbf{Q}}(\alpha)$. This character $\chi_{0}$ is called the modular character associated with $\chi$.

Notice that if $h_{\mathbf{L}}=1$ then $\chi=\nu \chi_{0}$ and, to compute $\chi$ on ideals, it remains to explain how one can effectively determine a generator $\alpha_{\mathcal{L}}$ of a principal primitive ideal

$$
\mathcal{L}=Q \mathbf{Z}+\frac{P+\sqrt{d_{\mathbf{L}}}}{2} \mathbf{Z}
$$

of norm $Q$. Set $x_{0}=\left(P+\sqrt{d_{\mathbf{L}}}\right) /(2 Q)$, let $x_{0}=\left[a_{0}, a_{1}, \cdots\right]$ be the continued fractional expansion of $x_{0}$, and set $x_{n}=\left[a_{n}, a_{n+1}, \cdots\right]$. It is well known that $x_{n}=$ $\left(P_{n}+\sqrt{d_{\mathbf{L}}}\right) /\left(2 Q_{n}\right)$, where the $P_{n}$ 's and $Q_{n}$ 's are recursively defined by

$$
\left\{\begin{array}{l}
a_{n}=\left[x_{n}\right]=\left[\left(P_{n}+\sqrt{d_{\mathbf{L}}}\right) /\left(2 Q_{n}\right)\right], \\
P_{n+1}=2 a_{n} Q_{n}-P_{n}, \\
Q_{n+1}=\left(d_{\mathbf{L}}-P_{n+1}^{2}\right) /\left(4 Q_{n}\right) .
\end{array}\right.
$$

We define two sequences $\left(p_{n}\right)_{n \geq-1}$ and $\left(q_{n}\right)_{n \geq-1}$ by setting

$$
\left\{\begin{array}{l}
\left(p_{-1}, q_{-1}\right)=(0,1), \\
\left(p_{0}, q_{0}\right)=(1,0), \\
\left(p_{n+1}, q_{n+1}\right)=\left(a_{n} p_{n}+p_{n-1}, a_{n} q_{n}+q_{n-1}\right) .
\end{array}\right.
$$


Since $\mathcal{L}$ is principal, there exists $m \geq 0$ such that $Q_{m}=1$ and

$$
\alpha_{\mathcal{L}}=\left(\left(2 p_{m} Q-q_{m} P\right)-q_{m} \sqrt{d_{\mathbf{L}}}\right) / 2
$$

is an explicit generator of the principal ideal $\mathcal{L}$ (see $[\mathrm{LO}]$ ).

Proposition 3 (See [Sie]). Choose $\gamma \in \mathbf{L}$ such that $\left(\gamma \sqrt{d_{\mathbf{L}}}\right) \mathcal{F}_{0}=\mathcal{I}$ is an integral ideal coprime with $\mathcal{F}_{0}$, and set

$$
\tau_{\mathcal{F}_{0}}\left(\gamma, \chi_{0}\right)=\sum_{\lambda \bmod \mathcal{F}_{0}} \chi_{0}(\lambda) e^{2 \pi i \operatorname{Tr}_{\mathbf{L} / \mathbf{Q}}(\lambda \gamma)}
$$

where $\operatorname{Tr}_{\mathbf{L} / \mathbf{Q}}$ stands for the trace from $\mathbf{L}$ to $\mathbf{Q}$. Then

$$
W_{\chi}=\chi(\mathcal{I}) \nu\left(\gamma \sqrt{d_{\mathbf{L}}}\right) \frac{\tau_{\mathcal{F}_{0}}\left(\gamma, \chi_{0}\right)}{\sqrt{N_{\mathbf{L} / \mathbf{Q}}\left(\mathcal{F}_{0}\right)}}
$$

has absolute value one and does not depend on the choice of $\gamma$. In particular, if $\mathcal{F}_{0}=(1)$ then we may take $\gamma=1 / \sqrt{d_{\mathbf{L}}}$, and we get $W_{\chi}=1$.

We now explain how to compute numerical approximations of $L(1, \chi)$ that are as good as desired for Hecke $L$-functions over real quadratic fields.

Theorem 4 (See Theorem 2 and (11)). 1. We have

$$
L(1, \chi)=\sum_{n \geq 1} \frac{a_{n}(\chi)}{n} K_{2,1}\left(n / A_{\chi}\right)+W_{\chi} \sum_{n \geq 1} \frac{\overline{a_{n}(\chi)}}{n} K_{2,2}\left(n / A_{\chi}\right),
$$

and since $0 \leq K_{2,2}(B) \leq K_{2,1}(B) \leq 2 e^{-B}$, these series are absolutely convergent.

2. For any positive integer $M \geq 2 A_{\chi}$, in setting

$$
S_{M}(\chi)=\sum_{n=1}^{M} \frac{a_{n}(\chi)}{n} K_{2,1}\left(n / A_{\chi}\right)+W_{\chi} \sum_{n=1}^{M} \frac{\overline{a_{n}(\chi)}}{n} K_{2,2}\left(n / A_{\chi}\right),
$$

we have

$$
\left|L(1, \chi)-S_{M}(\chi)\right| \leq 4(\log (M e)+1)^{2} e^{-M / A_{\chi}},
$$

and there exists $C_{1}>0$ absolute and effective such that for any $\mathbf{L}$, any $\chi$ and any $M \geq 0$ we have

and

$$
|L(1, \chi)| \leq C_{1} \log ^{2}\left(2 e A_{\chi}\right)
$$

$$
\left|S_{M}(\chi)\right| \leq C_{1} \log ^{2}\left(2 e A_{\chi}\right) .
$$

3. Conversely, there exists $C_{2}>0$ absolute and effective such that for any $\mathbf{L}$ and any non-quadratic $\chi$ we have

$$
|L(1, \chi)| \geq C_{2} / \log ^{2}\left(2 e A_{\chi}\right) .
$$

Proof. We prove point 2. We have $\left|a_{n}(\chi)\right| \leq d_{2}(n)$, where $d_{2}(n)$ is the number of factorizations of $n$ as a product of two positive integers. We now follow the same line of reasoning as in [Lou4], and set

$$
S(x) \stackrel{\text { def }}{=} \sum_{n \leq x} \frac{d_{2}(n)}{n} \leq\left(\sum_{n \leq x} \frac{1}{n}\right)^{2} \leq \log ^{2}(e x)
$$


and

$$
R_{M}(\chi)=\sum_{n>M} \frac{d_{2}(n)}{n} e^{-n / A_{\chi}}
$$

Using (11), we get $\left|L(1, \chi)-S_{M}(\chi)\right| \leq 4 R_{M}(\chi)$ and

$$
\begin{aligned}
4 R_{M}(\chi) & \leq 4 \sum_{n>M} S(n)\left(e^{-n / A_{\chi}}-e^{-(n+1) / A_{\chi}}\right) \\
& \leq \frac{4}{A_{\chi}} \sum_{n>M} \log ^{2}(e n) e^{-n / A_{\chi}} \\
& \leq \frac{4}{A_{\chi}} \int_{M}^{\infty} \log ^{2}(e x) e^{-x / A_{\chi}} d x=4 \int_{M / A_{\chi}}^{\infty} \log ^{2}\left(e A_{\chi} x\right) e^{-x} d x
\end{aligned}
$$

where the last inequality holds for $M$ such that $M \log (e M) \geq 2 A_{\chi}$, hence for $M \geq 2 A_{\chi}$ (note that we always have $A_{\chi} \geq 1 / 2$ ). Finally, in setting $a=M / A_{\chi} \geq 2$ and $b=e A_{\chi}>0$, we get (15) from

$$
\begin{aligned}
\int_{a}^{\infty} \log ^{2}(b x) e^{-x} d x & =e^{-a} \log ^{2}(a b)+2 \int_{a}^{\infty} \log (b x) e^{-x} \frac{d x}{x} \\
& \leq e^{-a} \log ^{2}(a b)+\frac{2}{a} \int_{a}^{\infty} \log (b x) e^{-x} d x \\
& =e^{-a} \log ^{2}(a b)+\frac{2}{a} e^{-a} \log (a b)+\frac{2}{a} \int_{a}^{\infty} e^{-x} \frac{d x}{x} \\
& \leq e^{-a} \log ^{2}(a b)+\frac{2}{a} e^{-a} \log (a b)+\frac{2}{a^{2}} e^{-a} .
\end{aligned}
$$

As for (16) and (17), we use the previous bound on $R_{M}(\chi)$ and note that

$$
4 \sum_{n \leq 2 A_{\chi}} \frac{d_{2}(n)}{n} e^{-n / A_{\chi}} \leq \frac{4}{A_{\chi}} \sum_{n \leq 2 A_{\chi}} \log ^{2}(e n) e^{-n / A_{\chi}} \leq 8 \log ^{2}\left(2 e A_{\chi}\right) .
$$

Finally, the proof of point 3 follows by the same method as in [Lou1].

Let $\mathbf{N}$ be a CM-field of degree $2 n$ which is an abelian extension of degree $n$ of some real quadratic subfield $\mathbf{L}$. Recall that

$$
h_{\mathbf{N}}^{-}=Q_{\mathbf{N}} w_{\mathbf{N}} \prod_{\chi \in X_{\mathbf{N} / \mathbf{L}}^{-}} \frac{A_{\chi}}{4 \pi} L(1, \chi)
$$

and set

$$
h_{\mathbf{N}}^{-}(M)=Q_{\mathbf{N}} w_{\mathbf{N}} \prod_{\chi \in X_{\mathbf{N} / \mathbf{L}}^{-}} \frac{A_{\chi}}{4 \pi} S_{\chi}(M),
$$

where the $S_{\chi}(M)$ are defined in Theorem 4 . When doing actual computations, to find an upper bound on $\left|h_{\overline{\mathbf{N}}}^{-}-h_{\mathbf{N}}^{-}(M)\right|$ we use (15)-(18) and the following technical lemma: 
Lemma 5. Let $\left(x_{k}\right)_{1 \leq k \leq n}$ be $n$ nonzero complex numbers, let $\left(\epsilon_{k}\right)_{1 \leq k \leq n}$ be $n$ complex numbers, and set $\epsilon \stackrel{\text { def }}{=} \max _{1 \leq k \leq n}\left(\left|\epsilon_{k}\right|\right)$. Then

$$
\left|\left(\prod_{k=1}^{n} x_{k}\right)-\left(\prod_{k=1}^{n}\left(x_{k}+\epsilon_{k}\right)\right)\right| \leq\left(\prod_{k=1}^{n}\left|x_{k}\right|\right)\left(\exp \left(\sum_{k=1}^{n} \epsilon /\left|x_{k}\right|\right)-1\right) .
$$

We refer the reader to Theorem 7 for a more general use of this technical lemma.

Now we explain how we can practically compute $K_{2,1}(B)$ and $K_{2,2}(B)$ for any $B>0$ :

Theorem 6. Let $\gamma=0.577215664901532860606512090 \cdots$ denote Euler's constant and let $B$ be positive. For $i \in\{1,2\}$, set

$$
a_{n, i}(B)=\gamma+\log B-\frac{1}{2 n+3-i}-\sum_{k=1}^{n} \frac{1}{k} .
$$

We have the following power series expansions:

$$
\begin{aligned}
& K_{2,1}(B)=1+4 \sum_{n \geq 0} a_{n, 1}(B) \frac{B^{2 n+2}}{(2 n+2)(n !)^{2}}, \\
& K_{2,2}(B)=\pi B+4 \sum_{n \geq 0} a_{n, 2}(B) \frac{B^{2 n+2}}{(2 n+1)(n !)^{2}}
\end{aligned}
$$

and for any integer $M \geq 0$ we have

$$
\left|\sum_{n>M} a_{n, i}(B) \frac{B^{2 n+2}}{(2 n+3-i)(n !)^{2}}\right| \leq \frac{2 B^{2 M+3}}{(M+1)(M !)^{2}} .
$$

Proof. For the sake of argument we only prove (20), and (21) for $i=2$. Recall that

$$
K_{2,2}(B)=\frac{1}{2 \pi i} \int_{\alpha-i \infty}^{\alpha+i \infty} \Gamma^{2}(s) \frac{B^{2-2 s}}{s-(1 / 2)} d s
$$

We set

$$
R_{M}=\frac{1}{2 \pi i} \int_{-M-\frac{1}{2}-i \infty}^{-M-\frac{1}{2}+i \infty} \Gamma^{2}(s) \frac{B^{2-2 s}}{s-(1 / 2)} d s
$$

and we notice that the poles of

$$
s \mapsto f(s)=\Gamma^{2}(s) B^{2-2 s} /(s-(1 / 2))
$$

are $s=1 / 2$ (which is a simple pole) and $s=-n$ for any $n \geq 0$ (which are double poles). Pushing the line of integration $\Re(s)=\alpha$ to the left to the line $\Re(s)=-M-\frac{1}{2}$, we obtain

$$
\begin{aligned}
K_{2,2}(B) & =\operatorname{Res}_{s=1 / 2}(f)+\sum_{n=0}^{M} \operatorname{Res}_{s=-n}(f)+R_{M} \\
& =\pi B-\sum_{n \geq 0}\left(\frac{1}{2 n+1}-\log B+\frac{\Gamma^{\prime}}{\Gamma}(n+1)\right) \frac{4 B^{2 n+2}}{(2 n+1)(n !)^{2}}+R_{M}
\end{aligned}
$$


Now, since $\left(\Gamma^{\prime} / \Gamma\right)(s+1)=\frac{1}{s}+\left(\Gamma^{\prime} / \Gamma\right)(s)$ and $\left(\Gamma^{\prime} / \Gamma\right)(1)=-\gamma$, we get $\left(\Gamma^{\prime} / \Gamma\right)(n+1)=$ $-\gamma+\sum_{k=1}^{n} \frac{1}{k}$. Finally, we noticed in [Lou6] that

$$
\left|\Gamma\left(-M-\frac{1}{2}+i t\right)\right|^{2}=\frac{\pi}{\cosh (\pi t)} \prod_{k=0}^{M}\left|\frac{2 k+1}{2}+i t\right|^{-2} \leq \frac{4 \pi}{(M !)^{2} \cosh (\pi t)},
$$

which yields the desired bound

$$
R_{M} \leq \frac{2 B^{2 M+3}}{(M+1)(M !)^{2}} \int_{-\infty}^{\infty} \frac{d t}{\cosh (\pi t)}=\frac{2 B^{2 M+3}}{(M+1)(M !)^{2}} .
$$

\section{EXAMPLES OF RELATIVE CLASS NUMBER COMPUTATIONS}

4.1. First example: Some Hilbert 2-class fields. We let $\mathbf{L}$ be a real quadratic field with fundamental unit of norm +1 and such that the 2-Sylow subgroup of its narrow ideal class group is cyclic of order $n=2^{m}$ for some $m \geq 2$ (and $h_{\text {odd }}$ will denote the odd part of the narrow class number of $\mathbf{L})$. Therefore, $\mathbf{L}=\mathbf{Q}\left(\sqrt{p_{1} p_{2}}\right)$ for some primes $p_{1}$ and $p_{2}$ not equal to 3 modulo 4 satisfying $2 \leq p_{1}<p_{2}$ and $\left(p_{1} / p_{2}\right)=+1$ (Legendre's symbol). We let $\mathbf{N}$ and $\mathbf{N}^{+}$denote the narrow Hilbert 2-class field and wide Hilbert 2-class field of $\mathbf{L}$, respectively. Then $\mathbf{N}$ is a dihedral CM-field of degree $2 n=2^{m+1} \geq 8$ with maximal totally real subfield $\mathbf{N}^{+}$. Moreover, the relative class number of a dihedral CM-field $\mathbf{N}$ of degree $2^{m+1} \geq 8$ is odd if and only if $\mathbf{N}$ is some such narrow Hilbert 2-class field (see [LO]). In that situation $Q_{\mathbf{N}}=w_{\mathbf{N}}=2, A_{\chi}=\sqrt{d_{\mathbf{L}} / \pi^{2}}$ and $W_{\chi}=1$ (use Proposition 3) do not depend on $\chi \in X_{\mathbf{N} / \mathbf{L}}^{-}$, and

$$
h_{\mathbf{N}}^{-}=\frac{4 d_{\mathbf{L}}^{n / 4}}{(2 \pi)^{n}} \prod_{\chi \in X_{\mathbf{N} / \mathbf{L}}^{-}} L(1, \chi)
$$

is a perfect square. Now we explain how we computed $a_{n}(\chi)$ on prime powers $n=p^{k}$ :

If $(p)=\mathcal{P}$ is inert in $\mathbf{L}$ then $\mathcal{P}$ is principal in the narrow sense, $\chi(\mathcal{P})=1$ and

$$
a_{p^{k}}(\chi)= \begin{cases}0 & \text { if } k \text { is odd } \\ 1 & \text { if } k \text { is even }\end{cases}
$$

Therefore, we have $a_{n}(\chi)=0$ if $\left(d_{\mathbf{L}} / n\right)=-1$ (Kronecker's symbol).

If $p \in\left\{p_{1}, p_{2}\right\}$ is ramified in $\mathbf{L}$, say $\mathcal{P}^{2}=(p)=\left(p_{i}\right)=\mathcal{P}_{i}^{2}$, then the order of $\mathcal{P}$ in the narrow ideal class group of $\mathbf{L}$ is equal to 1 or 2 , and $\chi(\mathcal{P})=1$ or -1 , respectively, and

$$
a_{p^{k}}(\chi)=\chi(\mathcal{P})^{k} .
$$

Note also that if $\mathcal{P}_{i}$ has order 1 in the narrow ideal class group of $\mathbf{L}$, then the other $\mathcal{P}_{j}$ has order 2 in the narrow ideal class group of $\mathbf{L}$ (for their product, which is equal to the principal ideal $\left(\sqrt{d_{\mathbf{L}}}\right)$, is principal in the wide sense but not principal in the narrow sense). In Table 1 , we let $p_{+}$denote the $p_{i}$ for which the prime ideal of $\mathbf{L}$ above $p_{i}$ is principal in the narrow sense. 
Finally, if $(p)=\mathcal{P} \mathcal{P}^{\prime}$ splits in $\mathbf{L}$, then $\chi\left(\mathcal{P}^{\prime}\right)=\chi(\mathcal{P})^{-1}$ and

$$
\begin{aligned}
a_{p^{k}}(\chi) & =\sum_{a=0}^{k} \chi\left(\mathcal{P}^{a}\right) \chi\left(\mathcal{P}^{\prime k-a}\right) \\
& = \begin{cases}k+1 & \text { if } \chi(\mathcal{P})=1, \\
\frac{(-1)^{k}(k+1)}{\sin \left(2(k+1) k_{\mathcal{P}} \pi / 2^{m}\right)} & \text { if } \chi(\mathcal{P})=-1, \\
\sin \left(2 k_{\mathcal{P}} \pi / 2^{m}\right) & \text { if } \chi(\mathcal{P})=\exp \left(2 k_{\mathcal{P}} \pi i / 2^{m}\right) \neq \pm 1 .\end{cases}
\end{aligned}
$$

We fix a prime ideal $\mathcal{Q}$ of $\mathbf{L}$ whose narrow ideal class has order $2^{m}$ in the narrow ideal class group of $\mathbf{L}$ (in practice, we choose $\mathcal{Q}$ whith smallest norm). Let $e_{p} \in$ $\left\{0,1, \cdots, 2^{m}-1\right\}$ be the only integer in this set such that $\mathcal{Q}^{e_{p}} \mathcal{P}^{h_{\text {odd }}}$ is principal in the narrow sense. Then, $\chi(\mathcal{P})=\chi(\mathcal{Q})^{-e_{p} h_{\text {odd }}^{\prime}}$ where $h_{\text {odd }} h_{\text {odd }}^{\prime} \equiv 1\left(\bmod 2^{m}\right)$. We also let $\psi$ be the character defined by $\psi(\mathcal{Q})=\exp \left(2 \pi i / 2^{m}\right)$. We have $X_{\mathbf{N} / \mathbf{L}}^{-}=$ $\left\{\psi^{i}, 0 \leq i \leq 2 n-1\right.$ and $i=2 j+1$ odd $\}$. Using the technique developed in this paper to compute the values at $s=1$ of these $n / 4=2 n / 8$ Hecke $L$-functions, we computed Table 1 of relative class numbers (the tables are in $\S 7$ ). It agrees with the results of the computations we did in [Lou3] and [LO]. In [LO] we used a technique peculiar to these Hilbert class fields developed in [Zag] to efficiently compute such relative class numbers. Let us point out that the use of Zagier's method requires the determination of the structure of the ideal class group of $\mathbf{L}$, whereas the method developed here does not require it, and, according to [LO, Table 2] and Table 1 of this paper, both techniques agree for the four Hilbert 2-class fields considered in [LO], for which the ideal class group of $\mathbf{L}$ is not cyclic.

4.2. Second example: Dihedral CM-fields of degree $4 p$. We now want to explain how we can use our previous results to efficiently compute relative class numbers of dihedral CM-fields $\mathbf{N}$. This was our main motivation for writing this paper. Indeed, in [LOO], to solve the class number one problem for the dihedral CM-fields $\mathbf{N}$ of degree 12 we reduced the computation of $h_{\mathbf{N}}^{-}$to that of $h_{\mathbf{N}_{0}}^{-}$, the relative class number of some non-normal sextic CM-subfield of $\mathbf{N}$, and we used the technique for computing relative class numbers of CM-fields developed in [Lou6]. But this technique is too slow to compute relative class numbers of dihedral CMfields $\mathbf{N}$ of higher degrees. Moreover, we used cubic polynomials $P_{\mathbf{K}}(X)$ defining the non-normal maximal real subfields $\mathbf{K}=\mathbf{N}_{0}^{+}$of such $\mathbf{N}_{0}$ 's to compute the coefficients $a_{n}\left(\chi_{\mathbf{N}_{0} / \mathbf{N}_{0}^{+}}\right)$. But it would be impossible to use such defining polynomials for fields of higher degrees, for their computation would be much too slow. To overcome both these problems we thought it more efficient to construct real dihedral CMfields $\mathbf{N}^{+}$of degree $2 p$ by constructing characters of order $p$ on ray class groups of real quadratic number fields $\mathbf{L}$, for this would then enable us to compute relative class numbers by using the technique developed in this paper.

Throughout this section, $\mathbf{N}$ will denote a dihedral CM-field of degree $4 p, p$ any odd prime; that is, $\mathbf{N}$ is a normal CM-field such the Galois group $\mathrm{Gal}(\mathbf{N} / \mathbf{Q})$ is the (non-abelian) dihedral group of order $4 p$. Since the complex conjugation must be in the center of this Galois group (see $[\mathrm{LOO}]$ ), then the maximal totally real subfield $\mathbf{N}^{+}$of $\mathbf{N}$ is normal, $\operatorname{Gal}\left(\mathbf{N}^{+} / \mathbf{Q}\right)$ is the dihedral group of order $2 p$ and we let $\mathbf{L}$ denote the only quadratic subfield of $\mathbf{N}$ over which $\mathbf{N}$ is cyclic. Therefore, $\mathbf{L}$ is real. We finally let $\mathbf{M}$ denote the maximal abelian subfield of $\mathbf{N}$. Therefore, $\mathbf{M}$ is an imaginary biquadratic bicyclic number field containing $\mathbf{L}$. Conversely, let $\mathbf{N}^{+}$ 
be a real dihedral number field of degree $2 p$, where $p \geq 3$ is an odd prime. Let $\mathbf{L}$ be the quadratic subfield of $\mathbf{N}^{+}$and let $\mathbf{M}$ be any imaginary biquadratic bicyclic field with maximal totally real subfield $\mathbf{L}$. Then $\mathbf{N}=\mathbf{M} \mathbf{N}^{+}$is a dihedral CM-field of degree $4 p$ which is cyclic over $\mathbf{L}$. It is known that there exist positive integers $f_{\mathbf{N}^{+} / \mathbf{L}}, f_{\mathbf{M} / \mathbf{L}}$ and $f_{\mathbf{N} / \mathbf{L}}$ such that $\mathcal{F}_{\mathbf{N}^{+} / \mathbf{L}}=\left(f_{\mathbf{N}^{+} / \mathbf{L}}\right), \mathcal{F}_{\mathbf{M} / \mathbf{L}}=\infty_{1} \infty_{2}\left(f_{\mathbf{M} / \mathbf{L}}\right)$ and $\mathcal{F}_{\mathbf{N} / \mathbf{L}}=\operatorname{lcm}\left(\mathcal{F}_{\mathbf{N}^{+} / \mathbf{L}}, \mathcal{F}_{\mathbf{M} / \mathbf{L}}\right)=\infty_{1} \infty_{2}\left(f_{\mathbf{N} / \mathbf{L}}\right)$ (see [Mar]). The following Theorem 7 and Proposition 9 explain how to compute relative class numbers of such dihedral CM-fields:

Theorem 7. Let $\mathbf{N}$ be a dihedral CM-field of degree $4 p$. Set $A_{\mathbf{N}}=\sqrt{d_{\mathbf{L}} f_{\mathbf{N} / \mathbf{L}}^{2} / \pi^{2}}$ and let $\chi$ be any one of the $p-1$ characters in $X_{\mathbf{N} / \mathbf{M}}^{-}=X_{\mathbf{N} / \mathbf{L}}^{-} \backslash X_{\mathbf{M} / \mathbf{L}}^{-}$.

1. $\chi=\chi_{+} \chi_{-}$, where $\chi_{+} \in X_{\mathbf{N}^{+} / \mathbf{L}}$ has order $p$, and $\chi_{-}=\chi_{\mathbf{M} / \mathbf{L}} \in X_{\mathbf{M} / \mathbf{L}}$, which does not depend on $\chi$, is the quadratic character associated with the quadratic extension $\mathbf{M} / \mathbf{L}$.

2. The character $\chi^{*}$ of $\mathrm{Gal}(\mathbf{N} / \mathbf{Q})$ induced by $\chi$ is a real valued irreducible character of degree 2 of the dihedral group $D_{4 p}$. Hence all the $a_{n}(\chi)$ are real and $L(1, \chi)>0, W_{\chi}=+1$ (see [FQ, Th. 1]) and $A_{\chi}=A_{\mathbf{N}}$ do not depend on $\chi$.

3. $Q_{\mathbf{N}}=Q_{\mathbf{M}}, w_{\mathbf{N}}=w_{\mathbf{M}}$ and $h_{\mathbf{M}}^{-}$divides $h_{\mathbf{N}}^{-}$(see $[\mathrm{LOO}]$ ), and $h_{\mathbf{N}}^{-} / h_{\mathbf{M}}^{-}=$ $\left(h_{\mathbf{N} / \mathbf{M}}^{-}\right)^{2}$ is a perfect square (see $[\mathrm{LP}]$ or the proof of Theorem 12) and

$$
h_{\mathbf{N} / \mathbf{M}}^{-}=\prod_{j=0}^{(p-3) / 2} \frac{A_{\mathbf{N}}}{4 \pi} L\left(1, \chi^{2 j+1}\right) .
$$

4. Let a real number $\lambda>1$ and a prime $p \geq 3$ be given. Set

$$
B^{\prime}(\mathbf{N})=\lambda \frac{p-1}{2} A_{\mathbf{N}} \log A_{\mathbf{N}}
$$

and

$$
h_{\mathbf{N} / \mathbf{M}}^{-}(M)=\prod_{j=0}^{(p-3) / 2} \frac{A_{\mathbf{N}}}{4 \pi} S_{M}\left(\chi^{2 j+1}\right),
$$

where the $S_{M}\left(\chi^{2 j+1}\right)$ 's are defined in Theorem 4. Assume that $M \geq B^{\prime}(\mathbf{N}) \geq$ $2 A_{\mathbf{M}}$. Then the limit $R(M)=\left|h_{\mathbf{N} / \mathbf{M}}^{-}-h_{\mathbf{N} / \mathbf{M}}^{-}(M)\right|$ as $A_{\mathbf{N}}$ approaches infinity is equal to 0 .

Proof. To prove point 3 we use (8) for $\mathbf{N}$ and $\mathbf{M}$ and notice that the characters in $X_{\mathbf{N} / \mathbf{M}}^{-}$come in conjugate pairs. Let us now prove point 4 . We simplify the notation and set $A=A_{\mathbf{N}}$. Since the $(p-1) / 2$ primitive characters which appear in (22) have the same order $2 p$, none of them is quadratic and we may use point 3 of Theorem 4. Set

$$
\epsilon_{M}=4(\log (e M)+1)^{2} e^{-M / A}
$$

(which decreases for $M \geq 2 A$ ). Using Lemma 5, (15) and (18), we obtain

$$
R(M) \leq C_{1}^{(p-1) / 2}\left(\frac{A}{4 \pi}\right)^{(p-1) / 2} \log ^{p-1}(2 e A)\left(\exp \left(\frac{p-1}{2 C_{2}} \epsilon_{M} \log ^{2}(2 e A)\right)-1\right) .
$$

Therefore, $M \geq B^{\prime}(\mathbf{N})$ yields $e^{-M / A} \leq A^{-\lambda(p-1) / 2}, \epsilon_{M} \ll \log ^{2}(2 e A) A^{-\lambda(p-1) / 2}$ and

from which the desired result follows.

$$
R(M) \ll \frac{\log ^{p+3}(2 e A)}{A^{(\lambda-1)(p-1) / 2}},
$$


Remark 8. When doing actual computation we use

$$
\prod_{j=0}^{(p-3) / 2} \frac{A_{\mathbf{N}}}{4 \pi}\left(S_{M}\left(\chi^{2 j+1}\right)-\epsilon_{M}\right) \leq h_{\mathbf{N} / \mathbf{M}}^{-} \leq \prod_{j=0}^{(p-3) / 2} \frac{A_{\mathbf{N}}}{4 \pi}\left(S_{M}\left(\chi^{2 j+1}\right)+\epsilon_{M}\right)
$$

(provided that all the $(p-1) / 2$ terms on the left hand side of these inequalites are positive), and we stop the computation when $M$ is large enough and such that these inequalities squeeze only one rational integer.

\section{Proposition 9.}

1. Let $\mathbf{L}_{0}$ and $\mathbf{L}_{1}$ denote the two imaginary quadratic subfields of $\mathbf{M}$. We have $f_{\mathbf{M} / \mathbf{L}}=\sqrt{d_{\mathbf{L}_{0}} d_{\mathbf{L}_{1}} / d_{\mathbf{L}}}$, and a rational prime $l$ divides $f_{\mathbf{M} / \mathbf{L}}$ if and only if it divides both $d_{\mathbf{L}_{0}}$ and $d_{\mathbf{L}_{1}}$. Now, since for any prime ideal $\mathcal{L}$ of $\mathbf{L}$ lying above some rational prime $l \geq 2$ the value $\chi_{\mathbf{M} / \mathbf{L}}(\mathcal{L})$ depends on $l$ only, we may define $\epsilon_{l}=\chi_{\mathbf{M} / \mathbf{L}}(\mathcal{L})$, and we have

$$
\epsilon_{l}= \begin{cases}0 & \text { if } \chi_{\mathbf{L}_{0}}(l)=\chi_{\mathbf{L}_{1}}(l)=0, \\ -1 & \text { if } \chi_{\mathbf{L}}(l) \neq-1 \text { and either } \chi_{\mathbf{L}_{0}}(l)=-1 \text { or } \chi_{\mathbf{L}_{1}}(l)=-1, \\ +1 & \text { otherwise }\end{cases}
$$

2. Let $\psi=\chi^{2 j+1}$ be any one of the $(p-1) / 2$ characters of order $2 p$ which appear in Theorem 7, and let $\psi=\psi_{+} \psi_{-}$with $\psi_{+} \in X_{\mathbf{N}^{+} / \mathbf{L}}$ and $\psi_{-}=\chi_{\mathbf{M} / \mathbf{L}}$ denote its factorization. Then, for any rational prime $l \geq 2$ we have:

(a) If l divides $f_{\mathbf{N} / \mathbf{L}}=\operatorname{lcm}\left(f_{\mathbf{N}^{+} / \mathbf{L}}, f_{\mathbf{M} / \mathbf{L}}\right)$, then $a_{l^{k}}(\psi)=0$.

(b) Assume that $l$ does not divide $f_{\mathbf{N} / \mathbf{L}}$.

(i) If $\chi_{\mathbf{L}}(l)=-1$, then $\epsilon_{l}=\psi_{+}(l)=+1$ and

$$
a_{l^{k}}(\psi)= \begin{cases}0 & \text { if } k \text { is odd }, \\ 1 & \text { if } k \text { is even. }\end{cases}
$$

In particular, $a_{n}(\psi)=0$ if $\chi_{\mathbf{L}}(n)=-1$.

(ii) If $\chi_{\mathbf{L}}(l)=0$, then $a_{l^{k}}(\psi)=\epsilon_{l}^{k}$.

(iii) If $\chi_{\mathbf{L}}(l)=+1$ and $(l)=\mathcal{L} \mathcal{L}^{\prime}$ in $L$, then

$$
a_{l^{k}}(\psi)= \begin{cases}(k+1) \epsilon_{l}^{k} & \text { if } \psi_{+}(\mathcal{L})=+1, \\ \frac{\sin \left(2(k+1) k_{\mathcal{L}} \pi / p\right)}{\sin \left(2 k_{\mathcal{L}} \pi / p\right)} \epsilon_{l}^{k} & \text { if } \psi_{+}(\mathcal{L})=\exp \left(2 k_{\mathcal{L}} \pi i / p\right) \neq+1 .\end{cases}
$$

In particular, if $a_{n}(\psi) \neq 0$, then $\operatorname{gcd}\left(n, f_{\mathbf{N} / \mathbf{L}}\right)=1$ and $\chi_{\mathbf{L}}(n) \neq-1$.

Proof. We only prove point 2. If $l$ not dividing $f_{\mathbf{N} / \mathbf{L}}$ is inert in $\mathbf{L}$, then $(l)=\mathcal{L}$ splits in $\mathbf{N}^{+} / \mathbf{L}$ and in $\mathbf{M} / \mathbf{L}$ (look at inertia fields), which yields $\psi_{+}(\mathcal{L})=+1$, $\psi_{-}(\mathcal{L})=+1$ and the desired result. If $l$ not dividing $f_{\mathbf{N} / \mathbf{L}}$ is ramified in $\mathbf{L}$ and $(l)=\mathcal{L}^{2}$, then $\mathcal{L}$ splits in $\mathbf{N}^{+} / \mathbf{L}$ (see [Mar, Prop. III.3 page 124]), $\psi_{+}(\mathcal{L})=+1$, and we get the desired result. Finally, assume that $l$ not dividing $f_{\mathbf{N} / \mathbf{L}}$ splits in $\mathbf{L}$, and write $(l)=\mathcal{L} \mathcal{L}^{\prime}$. Using the same line of reasoning as in [Cox, section $\mathrm{D}$ page 190-192], one can prove that any real dihedral field $\mathbf{N}^{+}$of degree $2 p$ and conductor $\mathcal{F}_{\mathbf{N}^{+} / \mathbf{L}}=\left(f_{\mathbf{N}^{+} / \mathbf{L}}\right)$ is a subfield of the ring class field of conductor $f_{\mathbf{N}^{+} / \mathbf{L}}$ of the real quadratic field $\mathbf{L}$ (see [Lef] or [LPL]). Hence, any $\psi_{+}$in $X_{\mathbf{N}^{+} / \mathbf{L}}$ must be trivial on the group generated by the principal ideals of the form $(\alpha)$, where 
$\alpha \in \mathbf{A}_{\mathbf{L}}$ satisfies $\alpha \equiv a\left(\bmod \left(f_{\mathbf{N}^{+} / \mathbf{L}}\right)\right)$ for some rational integer $a$ relatively prime to $f_{\mathbf{N}^{+} / \mathbf{L}}$. Therefore, we have $\psi_{+}((l))=+1$, which yields $\psi_{+}\left(\mathcal{L}^{\prime}\right)=\psi_{+}(\mathcal{L})^{-1}$,

$$
a_{l^{k}}(\psi)=\epsilon_{l}^{k} \sum_{a=0}^{k} \psi_{+}(\mathcal{L})^{a} \psi_{+}\left(\mathcal{L}^{\prime}\right)^{k-a}=\epsilon_{l}^{k} \psi_{+}(\mathcal{L})^{-k} \sum_{a=0}^{k} \psi_{+}(\mathcal{L})^{2 a},
$$

and the desired result.

It remains to explain how we can compute $\psi_{+}(\mathcal{L})$ and how we can construct real dihedral fields $\mathbf{N}^{+}$of degree $2 p$.

4.2.1. Numerical computation of the relative class numbers of some dihedral CMfields of degree $4 p$. To test the efficiency of our approach, we choose to apply it in the following simple situation: the computation of relative class numbers of dihedral CM-fields $\mathbf{N}$ of degree $4 p$ ( $p$ any odd prime) such that their real quadratic subfields $\mathbf{L}$ have class number one (which yields $\chi=\nu \chi_{0}$ ), such that the extensions $\mathbf{M} / \mathbf{L}$ are unramified at all the finite places (which yields $\mathcal{F}_{\mathbf{N} / \mathbf{L}}=\infty_{1} \infty_{2} \mathcal{F}_{\mathbf{N}^{+} / \mathbf{L}}$ and $\chi_{0}=\chi_{+, 0}$ for any $\chi \in X_{\mathbf{N} / \mathbf{L}}^{-}$) and such that the conductors $\mathcal{F}_{\mathbf{N}^{+} / \mathbf{L}}$ of the cyclic extensions $\mathbf{N}^{+} / \mathbf{L}$ of degree $p$ are as simple as possible, i.e. are of the form $\mathcal{F}_{\mathbf{N}^{+} / \mathbf{L}}=(q)$, where $q \neq p$ is a positive prime integer (see Point 1 of Proposition 10 below). We refer the reader to [LPL] for a comprehensive exposition of the construction and the computation of the relative class numbers of the dihedral CM-fields of degrees $4 p$ ( $p$ any odd prime). We also refer the reader to [Lef] for the use of the technique developed here in the determination of all the dihedral CM-fields with class number one. We collect in the following proposition all the information we need to construct such simple dihedral CM-fields and to compute their associated modular characters:

Proposition 10 (See [Mar], [Lef] and [LPL]). Let $p$ be a given odd prime. Let $\mathbf{L}$ be a given real quadratic field.

1. Let $\mathbf{N}^{+}$be a real dihedral field of degree $2 p$ containing $\mathbf{L}$. There exist distinct primes $q_{i}$ not equal to $p$ and satisfying $q_{i} \equiv \chi_{\mathbf{L}}\left(q_{i}\right)(\bmod p)$ such that

$$
f_{\mathbf{N}^{+} / \mathbf{L}}=p^{a} \prod_{i=1}^{r} q_{i}, \quad \text { where } a=0 \text { or } a= \begin{cases}2 & \text { if } p \text { does not divide } d_{L}, \\ 1 & \text { if } p>3 \text { divides } d_{L}, \\ 1 \text { or } 2 & \text { if } p=3 \text { divides } d_{L} .\end{cases}
$$

2. Assume that $p$ does not divide $h_{\mathbf{L}}$. There is a bijective correspondence between the real dihedral fields $\mathbf{N}^{+}$of degre $2 p$ containing $\mathbf{L}$ and of conductor $f_{+}>1$ and the groups of order $p$ generated by primitive characters of order $p$ on $\left(\mathbf{A}_{\mathbf{L}} /\left(f_{+}\right)\right)^{*}$ which are trivial on the image of $\mathbf{Z}$ and on the image of $\epsilon_{\mathbf{L}}$ in this group. In particular, if a prime $q \neq p$ is unramified in $\mathbf{L}$ and satisfies $q \equiv \chi_{\mathbf{L}}(q)(\bmod p)$, then there exists a real dihedral field $\mathbf{N}^{+}$of degree $2 p$ and conductor $q$ if and only if $p$ divides $\left(q-\chi_{\mathbf{L}}(q)\right) / n_{\mathbf{L}, q}$, where

$$
n_{\mathbf{L}, q}=\min \left\{n \geq 1 ; \exists k \in \mathbf{Z} \mid \epsilon_{\mathbf{L}}^{n} \equiv k \quad(\bmod (q))\right\},
$$

in which case such an $\mathbf{N}^{+}$is unique. Note that since the quotient group $\left(\mathbf{A}_{\mathbf{L}} /(q)\right)^{*} / \operatorname{Im} \mathbf{Z}$ has order $q-\chi_{\mathbf{L}}(q)$, then $n_{\mathbf{L}, q}$ always divides $q-\chi_{\mathbf{L}}(q)$.

3. Let $q \neq p$ be prime. 
(a) If $(q)=\mathcal{Q} \mathcal{Q}^{\prime}$ splits in $\mathbf{L}$ and $\phi$ is a nontrivial character on $\left(\mathbf{A}_{\mathbf{L}} /(q)\right)^{*}$ which is trivial on the image of $\mathbf{Z}$, then there exists a nontrivial character $\phi_{q}$ on $\left(\mathbf{A}_{\mathbf{L}} / \mathcal{Q}\right)^{*}$ such that for any $\alpha \in \mathbf{A}_{\mathbf{L}}$ prime to $(q)$ we have

$$
\phi(\alpha)=\phi_{q}\left(\alpha / \alpha^{\prime}\right)=\phi_{q}(\alpha) \overline{\phi_{q}\left(\alpha^{\prime}\right)},
$$

where $\alpha^{\prime}$ denotes the conjugate of $\alpha$ in $\mathbf{L}$. Since $(\mathbf{Z} / q \mathbf{Z})^{*}$ is canonically isomorphic to $\left(\mathbf{A}_{\mathbf{L}} / \mathcal{Q}\right)^{*}$, we may assume that $\phi_{q}$ is defined on $(\mathbf{Z} / q \mathbf{Z})^{*}$.

(b) If $(q)=\mathcal{Q}$ is inert in $\mathbf{L}$, then any character on $\left(\mathbf{A}_{\mathbf{L}} /(q)\right)^{*}$ of order $p$ dividing $q+1=q-\chi_{\mathbf{L}}(q)$ is necessarily trivial on the image of $\mathbf{Z}$.

In particular, if $\phi_{0}$ is a given character of order $p$ on the group $\left(\mathbf{A}_{\mathbf{L}} /(q)\right)^{*}$ which is trivial on the image of $\mathbf{Z}$ in this group, then $\left\{\phi_{0}^{k} ; 1 \leq k \leq p-1\right\}$ is the set of all the characters of order $p$ on the group $\left(\mathbf{A}_{\mathbf{L}} /(q)\right)^{*}$ which are trivial on the image of $\mathbf{Z}$.

Throughout the remainder of this section we will choose $\phi_{0}$ defined as follows:

1. Assume that $(q)=\mathcal{Q} \mathcal{Q}^{\prime}$ splits in $\mathbf{L}$. We let $g_{q}$ denote the least positive generator of $(\mathbf{Z} / q \mathbf{Z})^{*}$ and let $\phi_{q}$ be the character of order $p$ on $(\mathbf{Z} / q \mathbf{Z})^{*}$ defined by $\phi_{q}\left(g_{q}\right)=\exp (2 \pi i / p)$. If $P_{q} \in \mathbf{Z}$ is such that $4 q$ divides $d_{\mathbf{L}}-P_{q}^{2}$, then we may assume that

$$
\mathcal{Q}=q \mathbf{Z}+\frac{P_{q}+\sqrt{d_{\mathbf{L}}}}{2} \mathbf{Z} .
$$

It is easily checked that for any algebraic integer $\alpha=\left(x+y \sqrt{d_{\mathbf{L}}}\right) / 2 \in \mathbf{A}_{\mathbf{L}}$ we have

$$
\alpha \equiv \begin{cases}\left(x-y P_{q}\right) / 2 & (\bmod \mathcal{Q}) \\ \left(x+y P_{q}\right) / 2 & \left(\bmod \mathcal{Q}^{\prime}\right)\end{cases}
$$

and for any $\alpha \in\left(\mathbf{A}_{\mathbf{L}} /(q)\right)^{*}$ we can compute $n_{\alpha} \in \mathbf{Z}$ such that $n_{\alpha}=$ $\left(x-y P_{q}\right) /\left(x+y P_{q}\right)$ in $(\mathbf{Z} / q \mathbf{Z})^{*}$, and according to the previous lemma we set

$$
\phi_{0}(\alpha)=\exp \left(2 \pi j_{\alpha} i / p\right) \quad \text { where } j_{\alpha}=\min \left\{j \geq 0 ; n_{\alpha} \equiv g_{q}^{j} \quad(\bmod q)\right\} .
$$

2. Assume that $(q)=\mathcal{Q}$ is inert in $\mathbf{L}$. We let $g_{q}$ denote a generator of $\left(\mathbf{A}_{\mathbf{L}} /(q)\right)^{*}$ and according to the previous proposition we let $\phi_{0}$ be defined by $\phi_{0}\left(g_{q}\right)=$ $\exp (2 \pi i / p)$, which yields

$$
\phi_{0}(\alpha)=\exp \left(2 \pi j_{\alpha} i / p\right), \quad \text { where } j_{\alpha}=\min \left\{j \geq 0 ; \alpha \equiv g_{q}^{j} \quad(\bmod (q))\right\} .
$$

Now, in Point 2 of Proposition 9 we may assume that we have chosen $\chi$ such that $\chi_{+, 0}=\phi_{0}$. Hence, if $\psi=\chi^{2 j+1}$ is an in Point 2 of Proposition 9, then $\psi_{+}=\phi_{0}^{2 j+1}$, and we can now practically compute the coefficients $a_{l^{k}}\left(\chi^{2 j+1}\right)$ and the approximations $h_{\mathbf{N} / \mathbf{M}}^{-}(M)$ of $h_{\mathbf{N} / \mathbf{M}}^{-}$. In Table 2 the reader will find some examples of our computation of relative class numbers of such dihedral CM-fields of degree $4 p \geq 12$.

Remark 11. Take $\chi=\chi_{+} \chi_{-} \in X_{\mathbf{N} / \mathbf{L}}^{-}$and notice that since $\mathbf{M} / \mathbf{L}$ is assumed to be unramified at all the finite places, then $\chi_{0}=\chi_{+, 0}$ is a primitive character of order $p$ on $\left(\mathbf{A}_{\mathbf{L}} /(q)\right)^{*}$ which is trivial on the image of $\mathbf{Z}$ in this group. In Proposition 3 we choose $\gamma=\left(1 / q \sqrt{d_{\mathbf{L}}}\right)$, which yields $\mathcal{I}=\mathbf{A}_{\mathbf{L}}=(1)$ and

$$
W_{\chi}=\frac{1}{q} \tau_{(q)}\left(1 / q \sqrt{d_{\mathbf{L}}}, \chi_{0}\right)=\frac{1}{q} \sum_{\lambda} \chi_{(\bmod (q))}(\lambda) \exp \left(2 \pi i \operatorname{Tr}_{\mathbf{L} / \mathbf{Q}}\left(\lambda / q \sqrt{d_{\mathbf{L}}}\right)\right) .
$$


We used this formula to check numerically that all the Artin root numbers $W_{\chi}$ are indeed equal to +1 .

4.3. Third example: Quaternion octic CM-fields. Throughout this section we let $\mathbf{N}$ denote a normal octic CM-field whose Galois group is the quaternion group of order eight. In that situation, the maximal totally real subfield $\mathbf{N}^{+}$of $\mathbf{N}$ is a normal biquadratic bicyclic field, and we let $\mathbf{L}$ denote any one of the three real quadratic subfields of $\mathbf{N}^{+}$. Notice that $\mathbf{N} / \mathbf{L}$ is cyclic quartic, and we let $\chi$ denote either of the two conjugate quartic characters associated to this cyclic quartic extension $\mathbf{N} / \mathbf{L}$.

Theorem 12. Let $\mathbf{N}$ denote a quaternion octic CM-field. The character $\chi^{*}$ of $\operatorname{Gal}(\mathbf{N} / \mathbf{Q})$ induced by $\chi$ is the real valued character of degree two of the quaternion group. Therefore, since $L(s, \chi)=L(s, \chi, \mathbf{N} / \mathbf{L})=L\left(s, \chi^{*}, \mathbf{N} / \mathbf{Q}\right)$, then all the $a_{n}(\chi)$ are real, $W_{\chi}$ is equal either to +1 or to -1 , and $L(1, \chi)>0$. Finally, $Q_{\mathbf{N}}=1$, $w_{\mathbf{N}}=2, h_{\mathbf{N}}^{-}$is even (see [Lou5, Lemma 5$\left.]\right), h_{\mathbf{N}}^{-} / 2=\left(\tilde{h}_{\mathbf{N}}^{-}\right)^{2}$ is a perfect square and

$$
\tilde{h}_{\mathbf{N}}^{-}=\frac{A_{\chi}}{4 \pi} L(1, \chi)
$$

(use (8)). Let $\lambda>1$ be given, and set $B^{\prime}(\mathbf{N})=\lambda A_{\chi} \log A_{\chi}$ and

$$
\tilde{h}_{\mathbf{N}}^{-}(M) \stackrel{\text { def }}{=} \frac{A_{\chi}}{4 \pi} S_{M}(\chi)
$$

where the $S_{\chi}(M)$ are defined in Theorem 4. Assume that $M \geq B^{\prime}(\mathbf{N}) \geq 2 A_{\chi}$. Then the limit $R(M)=\left|\tilde{h}_{\mathbf{N}}^{-}-\tilde{h}_{\mathbf{N}}^{-}(M)\right|$ as $A_{\chi}$ approaches infinity is equal to 0 .

Proof. We have $h_{\mathbf{N}}^{-}=|L(0, \chi)|^{2} / 8$. Now, $\chi$ has order 4 and according to the SiegelKlingen theorem we have $L(0, \chi) \in \mathbf{Q}(i)$ (see [Hid, Cor. 1, page 57]), and since $\chi^{*}$ is real valued then $L(0, \chi)$ is real. Therefore, $L(0, \chi)$ is rational and the positive integer $h_{\mathbf{N}}^{-} / 2=|L(0, \chi)|^{2} / 16$, which is a square in $\mathbf{Q}$, is the square of some positive integer $\tilde{h}_{\mathbf{N}}^{-} \cdot \bullet$

Remark 13. Notice that the bound (1) is equal to $B(\mathbf{N})=\frac{\lambda^{2}}{4} A_{\chi}^{2} \log ^{2} A_{\chi}$.

4.3.1. Some pure quaternion octic CM-fields. We $\operatorname{set} \mathbf{L}=\mathbf{Q}(\sqrt{2})$ and let $\epsilon=1+\sqrt{2}$ denote the fundamental unit of the ring of algebraic integers $\mathbf{A}=\mathbf{Z}[\sqrt{2}]$ of $\mathbf{L}$. Let $q \equiv 3(\bmod 8)$ be a positive prime and let $\mathbf{N}_{q}$ denote the only pure quaternion octic CM-field with maximal totally real field $\mathbf{N}_{q}^{+}=\mathbf{Q}(\sqrt{2}, \sqrt{q})$, and notice that $\tilde{h}_{\mathbf{N}}^{-}$is odd if and only if $\mathbf{N}$ is some $\mathbf{N}_{q}$ (see [Fro] and [Lou5]). We have $d_{\mathbf{N}_{q}}=2^{24} q^{6}$ and $d_{\mathbf{N}_{q}^{+}}=2^{8} q^{2}$, so that we easily get $\mathcal{F}_{\mathbf{N} / \mathbf{L}}=\infty_{1} \infty_{2}(4 q \sqrt{2})$ and $\mathcal{F}_{\mathbf{N}^{+} / \mathbf{L}}=(2 q)$. Let $\chi \in X_{\mathbf{N} / \mathbf{L}}^{-}$denote any of the two conjugate characters of order 4 on the ray class group of conductor $\mathcal{F}_{\mathbf{N} / \mathbf{L}}$ of $\mathbf{L}$ associated to the cyclic quartic extension $\mathbf{N} / \mathbf{L}$. Then $A_{\chi}=16 q / \pi$.

Proposition $14\left(\right.$ See $[$ Fro] $)$. Let $q \equiv 3(\bmod 8)$ be a positive prime. Let $\mathcal{P}_{2}=$ $(\sqrt{2})$ denote the prime ramified ideal of $\mathbf{L}=\mathbf{Q}(\sqrt{2})$ lying above 2 . We may write $\chi=$ $\nu \chi_{0}$, where $\chi_{0}=\chi_{2} \chi_{q}$ is a primitive character of order 4 on the group $\left(\mathbf{A}_{\mathbf{L}} / \mathcal{F}_{q}\right)^{*}$ and where $\chi_{2}$ and $\chi_{q}$ are primitive quartic characters ont the groups $\left(\mathbf{A} / \mathcal{P}_{2}^{5}\right)^{*}$ and $(\mathbf{A} /(q))^{*}$, respectively.

1. We may assume that $\chi_{0}=\chi_{2} \chi_{q}$ is defined by means of $\chi_{2}(-1)=+1, \chi_{2}(5)=$ $-1, \chi_{2}(\epsilon)=\chi_{q}(\epsilon)=i=\exp (2 \pi i / 4)$. 
2. For any $\alpha \in \mathbf{A}_{\mathbf{L}}$ we have $\chi_{2}\left(\alpha^{\prime}\right)=\overline{\chi_{2}(\alpha)}, \chi_{q}\left(\alpha^{\prime}\right)=\overline{\chi_{q}(\alpha)}$ and $\chi_{0}\left(\alpha^{\prime}\right)=$ $\overline{\chi_{0}(\alpha)}$. In particular, if $(p)=\mathcal{P} \mathcal{P}^{\prime}$ splits in $\mathbf{L}$ then $\chi\left(\mathcal{P}^{\prime}\right)=\overline{\chi(\mathcal{P})}$

3. We have $a_{2^{m}}(\chi)=a_{q^{m}}(\chi)=0$, and if $p \neq 2$ and $p \neq q$ then we have

$$
a_{p^{m}}(\chi)= \begin{cases}0 & \text { if } p \text { is inert in } \mathbf{L} \text { and } m \text { is odd, } \\ \left(\chi((p))^{m / 2}\right. & \text { if } p \text { is inert in } \mathbf{L} \text { and } m \text { is even, } \\ (m+1) \epsilon_{p}^{m} & \text { if }(p)=\mathcal{P} \mathcal{P}^{\prime} \text { splits in } \mathbf{L} \text { and } \epsilon_{p}=\chi(\mathcal{P})= \pm 1 \\ \frac{1+(-1)^{m}}{2} \epsilon_{p}^{m} & \text { if }(p)=\mathcal{P} \mathcal{P}^{\prime} \text { splits in } \mathbf{L} \text { and } \epsilon_{p}=\chi(\mathcal{P})= \pm i .\end{cases}
$$

Hence, all the $a_{p^{m}}(\chi)$ are real.

4. We have $W_{\chi}=-1$.

Proof. We note that the multiplicative group

$$
\left(\mathbf{A}_{\mathbf{L}} / \mathcal{P}_{2}^{5}\right)^{*}=\left\{ \pm 5^{a} \epsilon^{b} ; a \in\{0,1\}, b \in\{0,1,2,3\}\right\}
$$

of order 16 is isomorphic to $(\mathbf{Z} / 2 \mathbf{Z}) \times(\mathbf{Z} / 2 \mathbf{Z}) \times(\mathbf{Z} / 4 \mathbf{Z})$, and that the multiplicative group $\left(\mathbf{A}_{\mathbf{L}} /(q)\right)^{*}$ of order $q^{2}-1 \equiv 8(\bmod 16)$ is cyclic. Now, as $\{1,5\}$ is the kernel of the canonical map $\left(\mathbf{A}_{\mathbf{L}} / \mathcal{P}_{2}^{5}\right)^{*} \longrightarrow\left(\mathbf{A}_{\mathbf{L}} / \mathcal{P}_{2}^{4}\right)^{*}$ and as $\chi_{2}$ is primitive, we must have $\chi_{2}(5)=-1$. As $(-1)^{\left(q^{2}-1\right) / 4}=1$, then -1 is a fourth power in $\left(\mathbf{A}_{\mathbf{L}} /(q)\right)^{*}$ and $\chi_{q}(-1)=+1$. Moreover, as $N_{\mathbf{L} / \mathbf{Q}}(\epsilon)=-1$ and $q \equiv 3(\bmod 4)$, then $\epsilon$ is not a square in $\left(\mathbf{A}_{\mathbf{L}} /(q)\right)^{*}$ and $\chi_{q}(\epsilon)= \pm i$. Finally, since

$$
1=\chi((1))=\left\{\begin{array}{l}
\chi((-1))=\nu(-1) \chi_{0}(-1)=\chi_{0}(-1)=\chi_{2}(-1) \chi_{q}(-1)=\chi_{2}(-1), \\
\chi\left((\epsilon)=\nu(\epsilon) \chi_{0}(\epsilon)=-\chi_{0}(\epsilon)=-\chi_{2}(\epsilon) \chi_{q}(\epsilon),\right.
\end{array}\right.
$$

we get $\chi_{2}(-1)=+1$ and $\chi_{q}(\epsilon)=\chi_{2}(\epsilon)= \pm i$. Now, $\chi_{2}\left(\epsilon^{\prime}\right)=\chi_{2}(-1 / \epsilon)=1 / \chi_{2}(\epsilon)=$ $\overline{\chi_{2}(\epsilon)}$ yields $\chi_{2}\left(\alpha^{\prime}\right)=\overline{\chi_{2}(\alpha)}$ for any $\alpha \in \mathbf{A}_{\mathbf{L}}$.

The following table is used to compute $\chi_{2}(\alpha)$ for $\alpha=x+y \sqrt{2} \in \mathbf{A}_{\mathbf{L}}$ prime to $\sqrt{2}$ :

\begin{tabular}{|l|lllllllll|}
\hline$\alpha \equiv$ & 1 & $\epsilon$ & $\epsilon^{2}$ & $\epsilon^{3}$ & -1 & $-\epsilon$ & $-\epsilon^{2}$ & $-\epsilon^{3}$ & $\left(\bmod \mathcal{P}_{2}^{5}\right)$ \\
$\alpha \equiv$ & 1 & $1+\sqrt{2}$ & $3+2 \sqrt{2}$ & $7+\sqrt{2}$ & 7 & $7+3 \sqrt{2}$ & $5+2 \sqrt{2}$ & $1+3 \sqrt{2}$ & $\left(\bmod \mathcal{P}_{2}^{5}\right)$ \\
$\chi_{2}(\alpha)$ & 1 & $i$ & -1 & $-i$ & 1 & $i$ & -1 & $-i$ & \\
\hline$\alpha \equiv$ & 5 & $5 \epsilon$ & $5 \epsilon^{2}$ & $5 \epsilon^{3}$ & -5 & $-5 \epsilon$ & $-5 \epsilon^{2}$ & $-5 \epsilon^{3}$ & $\left(\bmod \mathcal{P}_{2}^{5}\right)$ \\
$\alpha \equiv$ & 5 & $5+\sqrt{2}$ & $7+2 \sqrt{2}$ & $3+\sqrt{2}$ & 3 & $3+3 \sqrt{2}$ & $1+2 \sqrt{2}$ & $5+3 \sqrt{2}$ & $\left(\bmod \mathcal{P}_{2}^{5}\right)$ \\
$\chi_{2}(\alpha)$ & -1 & $-i$ & 1 & $i$ & -1 & $-i$ & 1 & $i$ & \\
\hline
\end{tabular}

Note that in setting $\eta_{q}=\epsilon^{\left(q^{2}-1\right) / 4}\left(\operatorname{modulo} \mathcal{P}_{2}^{5}\right)$, we have

$$
\chi_{q}(\alpha)=\exp \left(2 j_{\alpha} \pi i / 4\right)
$$

where

$$
j_{\alpha}=\min \left\{j \geq 0 ; \alpha^{\left(q^{2}-1\right) / 4} \equiv \eta_{q}^{j} \quad(\bmod (q))\right\} \in\{0,1,2,3\} .
$$

Of course, we use the binary representation of $\left(q^{2}-1\right) / 4$ to efficiently compute $\alpha^{\left(q^{2}-1\right) / 4}$ modulo the ideal $(q)$ of $\mathbf{L}$. To compute $\chi(\mathcal{P})$ we determine a generator $\alpha$ of $\mathcal{P}$ whih is known to be principal in $\mathbf{L}$, and we compute $\chi(\mathcal{P})=\chi((\alpha))=$ $\nu(\alpha) \chi_{2}(\alpha) \chi_{q}(\alpha)$. Numerical computations yield Table 3. We also computed several relative class numbers for $q$ large:

If $q=2 \cdot 10^{6}+3$, then $h_{\mathbf{N}_{q}}^{-}=851290664450=2 \cdot(652415)^{2}$.

If $q=10^{7}+19$, then $h_{\mathbf{N}_{q}}^{-}=66361628315682=2 \cdot(5760279)^{2}$. 
Remark 15. In Proposition 3 we choose $\gamma=1 /(16 q)$, which yields $\mathcal{I}=\mathbf{A}_{\mathbf{L}}=(1)$ and

$$
W_{\chi}=-\frac{1}{4 q \sqrt{2}} \sum_{x_{\lambda}+y_{\lambda} \sqrt{2}(\bmod (4 q \sqrt{2}))} \chi_{0}\left(x_{\lambda}+y_{\lambda} \sqrt{2}\right) \exp \left(4 \pi x_{\lambda} i / 16 q\right) .
$$

We used this formula to check numerically that all these Artin root numbers $W_{\chi}$ are indeed equal to -1 . Even checking this result yields a faster and more satisfactory method than the one used in [Lou2]. Note also that Shintani's method (see [Oka1]) is less efficient than the method developed here, for it would require us to compute $\gg q^{2}$ terms in some finite sum to compute the exact value of $h_{\mathbf{N}_{q}}^{-}$. Indeed, set

$$
B(x, y)=\frac{3}{2}\left(x^{2}+y^{2}-x-y+\frac{1}{3}\right)+\left(x-\frac{1}{2}\right)\left(y-\frac{1}{2}\right),
$$

let $\langle z\rangle$ denote the fractional part of the real number $z$, set

$$
\alpha_{a, b}=16 q\left\langle\frac{b}{16 q}\right\rangle+4 q\left(\left\langle\frac{4 a-3 b}{16 q}\right\rangle+3\left\langle\frac{b}{16 q}\right\rangle\right) \sqrt{2}
$$

and notice that $\mathbf{Z}[\sqrt{2}] \ni \alpha_{a, b} \equiv b+a \sqrt{2}(\bmod (4 q \sqrt{2}))$. Now, $h_{\mathbf{N}_{q}}^{-}=|L(0, \chi)|^{2} / 8$, and Shintani's method yields

$$
L(0, \chi)=\sum_{a=1}^{4 q} \sum_{\substack{b=1 \\ b \text { odd }}}^{16 q} \chi(b+a \sqrt{2}) B\left(\left\langle\frac{4 a-3 b}{16 q}\right\rangle,\left\langle\frac{b}{16 q}\right\rangle\right) .
$$

\section{Hecke $L$-FunCtions OVer totally REAL CUBiC FIELDS}

All the cases hitherto given refer to real quadratic fields $\mathbf{L}$. Therefore, we would like to finally use Theorem 2 on a simple example of a totally real cubic field $\mathbf{L}$. We choose $\mathbf{L}=\mathbf{Q}^{+}\left(\zeta_{7}\right)=\mathbf{Q}(\cos (2 \pi / 7))$ and set $\mathbf{N}=\mathbf{K L}$, where $\mathbf{K}$ denotes the imaginary cyclic quartic field $\mathbf{Q}\left(\zeta_{5}\right)$. Therefore, $\mathbf{N}$ is an imaginary cyclic field of degree $12, Q_{\mathbf{N}}=1, w_{\mathbf{N}}=10, \mathbf{N}^{+}=\mathbf{L}(\sqrt{5}), d_{\mathbf{L}}=7^{2}, d_{\mathbf{K}}=5^{3}, d_{\mathbf{N}^{+}}=5^{3} \cdot 7^{4}$, $d_{\mathbf{N}}=d_{\mathbf{K}}^{3} d_{\mathbf{L}}^{4}=5^{9} \cdot 7^{8}$ and $\mathcal{F}_{\mathbf{N} / \mathbf{L}}=\infty_{1} \infty_{2} \infty_{3}(5)$ (for 5 is inert in $\mathbf{L}$ ), and we must have $d_{\mathbf{N}}=d_{\mathbf{L}}^{4} N_{\mathbf{L} / \mathbf{Q}}\left(\mathcal{F}_{\mathbf{N} / \mathbf{L}}\right)$. Using [Wa], we can easily get $h_{\mathbf{N}}^{-}=1$. We now want to compute $h_{\mathbf{N}}^{-}$by using our technique for computing Hecke $L$-functions over totally real cubic fields at $s=1$. We let $\chi$ be either of the two conjugate quartic Hecke characters of conductor $\mathcal{F}_{\mathbf{N} / \mathbf{L}}$ associated with the cyclic quartic extension $\mathbf{N} / \mathbf{L}$. We have $A_{\chi}=\sqrt{7^{2} \cdot 5^{3} / \pi^{3}}$ and

$$
h_{\mathbf{N}}^{-}=\frac{7^{2} \cdot 5^{4}}{2^{5} \pi^{6}}|L(1, \chi)|^{2} .
$$

Moreover, as 5 is inert in $\mathbf{L} / \mathbf{Q}$, then $\left(\mathbf{A}_{\mathbf{L}} /(5)\right)^{*}$ is a cyclic group of order 124 and there are two characters of order 4 on this group, say $\chi_{0}$ and $\chi_{0}^{3}$. Let $\sigma$ be any of the generators of the cyclic Galois group $\operatorname{Gal}(\mathbf{L} / \mathbf{Q})$ of order three. Then $\chi_{0} \circ \sigma$ is necessarily equal to $\chi_{0}$ or $\chi_{0}^{3}$. But $\chi_{0} \circ \sigma=\chi_{0}^{3}$ would yield $\chi_{0}=\chi_{0} \circ \sigma^{3}=\chi_{0}^{27}=\chi_{0}^{3}$, which is impossible. Therefore, $\chi_{0} \circ \sigma=\chi_{0}$; and since for any integral ideal $\mathcal{I}$ of $\mathbf{A}_{\mathbf{L}}$ we have $\left(N_{\mathbf{L} / \mathbf{Q}}(\mathcal{I})\right)=\mathcal{I I}^{\sigma} \mathcal{I}^{\sigma^{2}}$ and since the narrow class number of $\mathbf{L}$ is equal to one, we get

$$
\chi(\mathcal{I})=\overline{\chi_{0}\left(N_{\mathbf{L} / \mathbf{Q}}(\mathcal{I})\right)}
$$

and 
Lemma 16. We have $a_{5^{m}}(\chi)=0$, and if $p \neq 5$ then

$$
a_{p^{m}}(\chi)=\left\{\begin{array}{lll}
\bar{\chi}_{0}^{m}(p) & \text { if } p=7, \\
\frac{(m+1)(m+2)}{2} \bar{\chi}_{0}^{m}(p) & \text { if } p \equiv \pm 1 \quad(\bmod 7) \quad(\text { and } p \neq 7) \\
0 & \text { if } p \neq \pm 1 \quad(\bmod 7) \text { and } 3 \nmid m(\text { and } p \neq 7) \\
\bar{\chi}_{0}^{m}(p) & \text { if } p \neq \pm 1 \quad(\bmod 7) \text { and } 3 \mid m(\text { and } p \neq 7)
\end{array}\right.
$$

Note that the restriction of $\chi_{0}$ to the image of $\mathbf{Z}$ in $\left(\mathbf{A}_{\mathbf{L}} /(5)\right)^{*}$ must be a quartic character. Therefore, we may assume that this restriction is defined by the following table, which enables us to compute $\chi_{0}(p)$ for any prime $p \neq 5$ :

$$
\begin{array}{|l|rrrr|}
\hline n & 1 & 2 & 3 & 4 \\
\hline \chi_{0}(n) & 1 & i & -i & -1 \\
\hline
\end{array}
$$

Now, as $\mathbf{L}$ is a totally real cubic field, the proof of Theorem 6 yields:

Theorem 17. Let $\gamma=0.577215664901532860606512090 \cdots$ denote Euler's constant and let $B$ be positive. Set $s_{1}(0)=-\gamma, s_{2}(0)=\pi^{2} / 6$, and for $n \geq 1$ set

$$
s_{1}(n)=-\gamma+\sum_{k=1}^{n} \frac{1}{k}, \quad s_{2}(n)=\frac{\pi^{2}}{6}+\sum_{k=1}^{n} \frac{1}{k^{2}} .
$$

and for $i \in\{1,2\}$, set $a_{n, i}(B)=A_{n, i}-B_{n, i} \log B+4 \log ^{2} B$, where

$$
A_{n, i}=\frac{8}{(2 n+3-i)^{2}}+\frac{12 s_{1}(n)}{2 n+3-i}+9\left(s_{1}(n)\right)^{2}+3 s_{2}(n)
$$

and

$$
B_{n, i}=\frac{8}{2 n+3-i}+12 s_{1}(n) .
$$

We have the following power series expansions:

$$
K_{3,1}(B)=1+\sum_{n \geq 0} a_{n, 1}(B) \frac{\left(-B^{2}\right)^{n+1}}{(2 n+2)(n !)^{3}}
$$

and

$$
K_{3,2}(B)=\pi^{3 / 2} B+\sum_{n \geq 0} a_{n, 2}(B) \frac{\left(-B^{2}\right)^{n+1}}{(2 n+1)(n !)^{3}} .
$$

Finally, we explain on this particular example a general strategy based on (12) which enables us to compute $W_{\chi}$ efficiently. We use the results of Section 2: we set

$$
S=\sum_{n \geq 1} a_{n}(\chi) H_{3}(n / A)
$$

and note that

$$
H_{3}(B)=-K_{3,1}^{\prime}(B)=B \sum_{n \geq 0} h_{n} \frac{\left(-B^{2}\right)^{n}}{(n !)^{3}}
$$

with

$$
h_{n}=9\left(s_{1}(n)\right)^{2}+3 s_{2}(n)-12 s_{1}(n) \log B+4 \log ^{2} B,
$$

which enables us to compute numerical approximations of $S$ as precisely as desired. If we can deduce from them that $S \neq 0$, then on plugging $x=1$ in (12) we get $W_{\chi}=$ $S / \bar{S}$, which enables us to use (10) for numerical computations. Note that if $W_{\chi}=$ -1 then necessarily $S=0$, so that this trick is useless for pure quaternion octic 
CM-fields. However, in our present situation, according to numerical computation, we do have $S \neq 0$, and we computed

$$
\begin{gathered}
S=1.093107 \cdots+0.998977 \cdots i, \\
W_{\chi}=0.089805 \cdots+0.995959 \cdots i, \\
L(1, \chi)=0.975462 \cdots+0.230275 \cdots i
\end{gathered}
$$

and $h_{\mathbf{N}}^{-}=1$. Moreover, numerical computations suggest, and one can prove, that

$$
W_{\chi}^{4}=(117-44 i) / 125=(11-2 i)^{2} / 125 .
$$

\section{FinAl REMARKS}

We refer the reader to [Lou7] for an efficient technique for computing relative class numbers of abelian CM-fields by using a technique similar to the one developed here for evaluating $L$-series associated with Dirichlet characters at $s=1$.

The reader will find in [Lou7] an extensive use of the technique introduced in section 5 for computing Artin root numbers $W_{\chi}$. We also point out that the example dealt with in Section 5 is not satisfactory, and that it would be worth computing relative class numbers of CM-fields of degree greater than six which would be neither abelian nor abelian extension of real quadratic fields, but which would be abelian extensions of totally real cubic fields.

Added in February 1999: We can now refer the reader to [S. Louboutin, Computation of $L(0, \chi)$ and of related class numbers of $C M$-fields, Preprint Univ. Caen (1999), submitted] for more satisfactory examples. The reader will find there examples of computation of relative class numbers of non-abelian normal CM-fields $\mathbf{N}$ of degree 24 and Galois group the special linear group over the finite field with three elements by using evaluations of $L(1, \chi)$ for Hecke $L$-functions associated with quartic characters $\chi$ on narrow ray class groups of non-normal totally real sextic fields $\mathbf{L}$.

We refer the reader to [LP] for an application of the technique developed here to the computation of relative class numbers of dicyclic CM-fields of degree $4 p$. Let us point out that, in contrast with dihedral CM-fields of degree $4 p$, where Artin root

numbers $W_{\chi}$ are always equal to +1 , Artin root numbers $W_{\chi}$ of dicyclic CM-fields of degree $4 p$ may depend on $\chi$.

All our computations were programmed in Kida's language Ubasic, which allows fast arbitrary precision calculation on PC's. 


\section{TABLES}

TABle 1. Some Hilbert 2-class fields

\begin{tabular}{|c|c|c|c|c|c|c|}
\hline$d_{\mathbf{L}}$ & $p_{+}$ & $h_{\mathbf{L}}$ & $\mathcal{Q}$ & $2 n$ & & $h_{\mathbf{N}}^{-}$ \\
\hline 776 & 2 & 2 & $5 \mathbf{Z}+\frac{6+\sqrt{d_{\mathbf{L}}}}{2} \mathbf{Z}$ & 8 & $L(1, \psi)=2.215788$ & $9=3^{2}$ \\
\hline 2005 & 5 & 4 & $3 \mathbf{Z}+\frac{1+\sqrt{d_{\mathbf{L}}}}{2} \mathbf{Z}$ & 16 & $\begin{array}{l}L(1, \psi)=2.386755 \\
L\left(1, \psi^{3}\right)=1.139895\end{array}$ & $49=7^{2}$ \\
\hline 1488392 & 2 & 36 & $83 \mathbf{Z}+\frac{160+\sqrt{d_{\mathbf{L}}}}{2} \mathbf{Z}$ & 16 & $\begin{array}{l}L(1, \psi)=2.527962 \\
L\left(1, \psi^{3}\right)=2.390673\end{array}$ & $11543^{2}$ \\
\hline 2234773 & 5573 & 36 & $53 \mathbf{Z}+\frac{97+\sqrt{d_{\mathbf{L}}}}{2} \mathbf{Z}$ & 16 & $\begin{array}{l}L(1, \psi)=1.637874 \\
L\left(1, \psi^{3}\right)=1.002971\end{array}$ & $\begin{array}{l}22193521 \\
=4711^{2}\end{array}$ \\
\hline 2331641 & 13 & 36 & $109 \mathbf{Z}+\frac{135+\sqrt{d_{\mathbf{L}}}}{2} \mathbf{Z}$ & 16 & $\begin{array}{l}L(1, \psi)=2.619148 \\
L\left(1, \psi^{3}\right)=0.535047\end{array}$ & $\begin{array}{l}17581249 \\
=4193^{2}\end{array}$ \\
\hline 5249 & 29 & 8 & $2 \mathbf{Z}+\frac{1+\sqrt{d_{\mathbf{L}}}}{2} \mathbf{Z}$ & 32 & $\begin{array}{l}L(1, \psi)=4.124139 \\
L\left(1, \psi^{3}\right)=0.986256 \\
L\left(1, \psi^{5}\right)=0.741952 \\
L\left(1, \psi^{7}\right)=0.686522\end{array}$ & $2209=47^{2}$ \\
\hline 2908841 & 223757 & 72 & $307 \mathbf{Z}+\frac{311+\sqrt{d_{\mathbf{L}}}}{2} \mathbf{Z}$ & 32 & $\begin{array}{l}L(1, \psi)=0.510605 \\
L\left(1, \psi^{3}\right)=0.802548 \\
L\left(1, \psi^{5}\right)=0.807890 \\
L\left(1, \psi^{7}\right)=3.063943\end{array}$ & $7066769^{2}$ \\
\hline 28981 & 7 & 16 & $5 \mathbf{Z}+\frac{1+\sqrt{d_{\mathbf{L}}}}{2} \mathbf{Z}$ & 64 & $\begin{array}{l}L(1, \psi)=2.852337 \\
L\left(1, \psi^{3}\right)=1.492483 \\
L\left(1, \psi^{5}\right)=1.433773 \\
L\left(1, \psi^{7}\right)=2.964050 \\
L\left(1, \psi^{9}\right)=1.594397 \\
L\left(1, \psi^{11}\right)=0.501012 \\
L\left(1, \psi^{13}\right)=0.442302 \\
L\left(1, \psi^{15}\right)=1.706111\end{array}$ & $2607679^{2}$ \\
\hline
\end{tabular}


TABLE 2. Some dihedral CM-fields of degree $4 p$

\begin{tabular}{|c|c|c|c|c|c|c|c|}
\hline$p$ & $4 p$ & $d_{\mathbf{L}}$ & $q$ & $\chi_{\mathbf{L}}(q)$ & M & all $W_{\psi^{2 j+1}}=1$ and & $h_{\mathbf{M}}^{-}=1$ and $h_{\mathbf{N}}^{-}=$ \\
\hline 3 & 12 & 21 & 109 & +1 & $\mathbf{Q}(\sqrt{-3}, \sqrt{-7})$ & $L(1, \psi)=1.422643$ & $324=18^{2}$ \\
\hline 3 & 12 & 21 & 23 & -1 & $\mathbf{Q}(\sqrt{-3}, \sqrt{-7})$ & $L(1, \psi)=1.498242$ & $16=4^{2}$ \\
\hline 5 & 20 & 21 & 101 & +1 & $\mathbf{Q}(\sqrt{-3}, \sqrt{-7})$ & $\begin{array}{l}L(1, \psi)=1.068576 \\
L\left(1, \psi^{3}\right)=1.831487\end{array}$ & $72361=269^{2}$ \\
\hline 5 & 20 & 21 & 139 & -1 & $\mathbf{Q}(\sqrt{-3}, \sqrt{-7})$ & $\begin{array}{l}L(1, \psi)=1.740963 \\
L\left(1, \psi^{3}\right)=1.048031\end{array}$ & $225625=475^{2}$ \\
\hline 7 & 28 & 57 & 43 & +1 & $\mathbf{Q}(\sqrt{-3}, \sqrt{-19})$ & $\begin{array}{l}L(1, \psi)=0.818558 \\
L\left(1, \psi^{3}\right)=0.531292 \\
L\left(1, \psi^{5}\right)=2.055108\end{array}$ & $247009=497^{2}$ \\
\hline 7 & 28 & 69 & 97 & -1 & $\mathbf{Q}(\sqrt{-3}, \sqrt{-23})$ & $\begin{array}{l}L(1, \psi)=3.122215 \\
L\left(1, \psi^{3}\right)=2.822641 \\
L\left(1, \psi^{5}\right)=1.943550\end{array}$ & $\begin{array}{l}21205475641 \\
=145621^{2}\end{array}$ \\
\hline 11 & 44 & 209 & 23 & +1 & $\mathbf{Q}(\sqrt{-11}, \sqrt{-19})$ & $\begin{array}{l}L(1, \psi)=0.450594 \\
L\left(1, \psi^{3}\right)=1.863407 \\
L\left(1, \psi^{5}\right)=4.914253 \\
L\left(1, \psi^{7}\right)=1.324753 \\
L\left(1, \psi^{9}\right)=0.589165\end{array}$ & $\begin{array}{l}18631977001 \\
=136499^{2}\end{array}$ \\
\hline 11 & 44 & 209 & 263 & -1 & $\mathbf{Q}(\sqrt{-11}, \sqrt{-19})$ & $\begin{array}{l}L(1, \psi)=0.580602 \\
L\left(1, \psi^{3}\right)=4.658983 \\
L\left(1, \psi^{5}\right)=0.502571 \\
L\left(1, \psi^{7}\right)=1.229407 \\
L\left(1, \psi^{9}\right)=1.594568\end{array}$ & $22082695283^{2}$ \\
\hline 13 & 52 & 209 & 157 & +1 & $\mathbf{Q}(\sqrt{-11}, \sqrt{-19})$ & $\begin{array}{l}L(1, \psi)=1.541778 \\
L\left(1, \psi^{3}\right)=1.115754 \\
L\left(1, \psi^{5}\right)=0.652133 \\
L\left(1, \psi^{7}\right)=3.103389 \\
L\left(1, \psi^{9}\right)=0.391887 \\
L\left(1, \psi^{11}\right)=3.370253\end{array}$ & $166060224707^{2}$ \\
\hline 17 & 68 & 57 & 101 & -1 & $\mathbf{Q}(\sqrt{-3}, \sqrt{-19})$ & $\begin{array}{l}L(1, \psi)=0.885015 \\
L\left(1, \psi^{3}\right)=0.381026 \\
L\left(1, \psi^{5}\right)=3.457565 \\
L\left(1, \psi^{7}\right)=0.342002 \\
L\left(1, \psi^{9}\right)=1.705920 \\
L\left(1, \psi^{11}\right)=0.553422 \\
L\left(1, \psi^{13}\right)=0.571116 \\
L\left(1, \psi^{15}\right)=1.785428\end{array}$ & $7436708089^{2}$ \\
\hline
\end{tabular}


TABle 3. The 50 pure quaternion octic CM-fields with $q \leq 1171$.

\begin{tabular}{|c|c|c|c|c|c|c|c|}
\hline \multicolumn{8}{|c|}{ All $W_{\chi}=-1$ and } \\
\hline case & $q$ & $L(1, \chi)$ & $h_{\mathbf{N}_{q}}^{-}=2\left(\hat{h}_{\mathbf{N}_{q}}^{-}\right)^{2}$ & case & $q$ & $L(1, \chi)$ & $h_{\mathbf{N}_{q}}^{-}=2\left(h_{\mathbf{N}_{q}}^{-}\right)^{2}$ \\
\hline 1 & 3 & 0.822467 & 2 & 26 & 523 & 0.693514 & $43218=2 \cdot 147^{2}$ \\
\hline 2 & 11 & 0.672927 & $18=2 \cdot 3^{2}$ & 27 & 547 & 0.825474 & $66978=2 \cdot 183^{2}$ \\
\hline 3 & 19 & 0.909042 & $98=2 \cdot 7^{2}$ & 28 & 563 & 0.714363 & $53138=2 \cdot 163^{2}$ \\
\hline 4 & 43 & 0.516432 & $162=2 \cdot 9^{2}$ & 29 & 571 & 0.635215 & $43218=2 \cdot 147^{2}$ \\
\hline 5 & 59 & 0.878227 & $882=2 \cdot 21^{2}$ & 30 & 587 & 1.021428 & $118098=2 \cdot 243^{2}$ \\
\hline 6 & 67 & 0.994325 & $1458=2 \cdot 27^{2}$ & 31 & 619 & 0.777291 & $76050=2 \cdot 195^{2}$ \\
\hline 7 & 83 & 0.743193 & $1250=2 \cdot 25^{2}$ & 32 & 643 & 0.732929 & $72962=2 \cdot 191^{2}$ \\
\hline 8 & 107 & 1.176050 & $5202=2 \cdot 51^{2}$ & 33 & 659 & 0.677692 & $65522=2 \cdot 181^{2}$ \\
\hline 9 & 131 & 0.960591 & $5202=2 \cdot 51^{2}$ & 34 & 683 & 1.304146 & $260642=2 \cdot 361^{2}$ \\
\hline 10 & 139 & 0.940807 & $5618=2 \cdot 53^{2}$ & 35 & 691 & 0.789139 & $97682=2 \cdot 221^{2}$ \\
\hline 11 & 163 & 0.560084 & $2738=2 \cdot 37^{2}$ & 36 & 739 & 0.724527 & $94178=2 \cdot 217^{2}$ \\
\hline 12 & 179 & 1.171670 & $14450=2 \cdot 85^{2}$ & 37 & 787 & 0.868449 & $153458=2 \cdot 277^{2}$ \\
\hline 13 & 211 & 1.321404 & $25538=2 \cdot 113^{2}$ & 38 & 811 & 1.104397 & $263538=2 \cdot 363^{2}$ \\
\hline 14 & 227 & 0.923916 & $14450=2 \cdot 85^{2}$ & 39 & 827 & 1.202373 & $324818=2 \cdot 403^{2}$ \\
\hline 15 & 251 & 0.756931 & $11858=2 \cdot 77^{2}$ & 40 & 859 & 0.692251 & $116162=2 \cdot 241^{2}$ \\
\hline 16 & 283 & 0.898029 & $21218=2 \cdot 103^{2}$ & 41 & 883 & 1.193182 & $364658=2 \cdot 427^{2}$ \\
\hline 17 & 307 & 0.892122 & $24642=2 \cdot 111^{2}$ & 42 & 907 & 1.341156 & $486098=2 \cdot 493^{2}$ \\
\hline 18 & 331 & 0.618713 & $13778=2 \cdot 83^{2}$ & 43 & 947 & 0.612290 & $110450=2 \cdot 235^{2}$ \\
\hline 19 & 347 & 0.604406 & $14450=2 \cdot 85^{2}$ & 44 & 971 & 0.663225 & $136242=2 \cdot 261^{2}$ \\
\hline 20 & 379 & 0.761704 & $27378=2 \cdot 117^{2}$ & 45 & 1019 & 1.324502 & $598418=2 \cdot 547^{2}$ \\
\hline 21 & 419 & 0.818541 & $38642=2 \cdot 139^{2}$ & 46 & 1051 & 0.903852 & $296450=2 \cdot 385^{2}$ \\
\hline 22 & 443 & 1.019265 & $66978=2 \cdot 183^{2}$ & 47 & 1091 & 0.915946 & $328050=2 \cdot 405^{2}$ \\
\hline 23 & 467 & 0.966883 & $66978=2 \cdot 183^{2}$ & 48 & 1123 & 0.845903 & $296450=2 \cdot 385^{2}$ \\
\hline 24 & 491 & 1.211086 & $116162=2 \cdot 241^{2}$ & 49 & 1163 & 1.232639 & $675122=2 \cdot 581^{2}$ \\
\hline 25 & 499 & 1.152113 & $108578=2 \cdot 233^{2}$ & 50 & 1171 & 1.262146 & $717602=2 \cdot 599^{2}$ \\
\hline
\end{tabular}

\section{AdDED AFter Posting}

In the statement after Theorem 17, the sentence that reads

"Note that if $W_{\chi}=-1$ then necessarily ..."

should read

"Note that if $S$ is real and $W_{\chi}=-1$ then necessarily ...". 


\section{REFERENCES}

[Cox] D. A. Cox. Primes of the form $x^{2}+n y^{2}$. John Wiley\&Sons, 1989. MR 90m:11016

[FQ] A. Fröhlich and J. Queyrut. On the functional equation of the Artin $L$-function for characters of real representations. Inventiones math. 20 (1973), 125-138. MR 48:253

[Fro] A. Fröhlich. Artin root numbers and normal integral bases for quaternion fields. Invent. math. 17 (1972), 143-166. MR 48:2115

[Hid] H. Hida. Elementary theory of L-functions and Eisenstein series. London Mathematical Society, Student Texts 26, Cambridge University Press, 1993. MR 94j:11044

[Lef] Y. Lefeuvre. Corps diédraux à multiplication complexe principaux. Preprint Univ. Caen (1997)

[Lou1] S. Louboutin. Minoration au point 1 des fonctions $L$ et détermination des corps sextiques abéliens totalement imaginaires principaux. Acta Arith. 72 (1992), 109-124. MR 93h: 11100

[Lou2] S. Louboutin. Calcul des nombres de classes relatifs : application aux corps octiques quaternioniques à multiplication complexe. C. R. Acad. Sci. Paris 317 (1993), 643-646. MR 94j:11111

[Lou3] S. Louboutin. Calcul des nombres de classes relatifs de certains corps de classes de Hilbert. C. R. Acad. Sci. Paris 319 (1994), 321-325. MR 95g:11111

[Lou4] S. Louboutin. Calcul du nombre de classes des corps de nombres. Pacific J. Math. 171 (1995), 455-467. MR 97a:11176

[Lou5] S. Louboutin. Determination of all quaternion octic CM-fields with class number 2. J. London Math. Soc. 54 (1996), 227-238. MR 97g:11122

[Lou6] S. Louboutin. Computation of relative class numbers of CM-fields. Math. Comp. 66 (1997), 1185-1194. MR 97k:11157

[Lou7] S. Louboutin. Computation of relative class numbers of imaginary abelian number fields. Exp. Math. 7 (1998), 293-303.

[LO] S. Louboutin and R. Okazaki. The class number one problem for some non-abelian normal CM-fields of 2-power degees. Proc. London Math. Soc. (3) 76 (1998), 523-548. CMP 98:11

[LOO] S. Louboutin, R. Okazaki and M. Olivier. The class number one problem for some non-abelian normal CM-fields. Trans. Amer. Math. Soc. 349 (1997), 3657-3678. MR 97k:11149

[LP] S. Louboutin and Y.-H. Park. Class number problems for dicyclic CM-fields. Acta Arith., to appear.

[LPL] S. Louboutin, Y.-H. Park and Y. Lefeuvre. Construction of the real dihedral number fields of degree $2 p$. Applications. Acta Arith. (to appear).

[Mar] J. Martinet. Sur l'arithmétique des extensions à groupe de Galois diédral d'ordre $2 p .$. Ann. Inst. Fourier (Grenoble) 19 (1969), 1-80. MR 41:6820

[Mey] C. Meyer. Die Berechnung der Klassenzahl abelscher Körper über quadratischen Zahlkörpern. Akademie-Verlag. Berlin, 1957. MR 19:531f

[Oka1] R. Okazaki. On evaluation of $L$-functions over real quadratic fields. J. Math. Kyoto Univ. 31 (1991), 1125-1153. MR 93b:11154

[Oka2] R. Okazaki. An elementary proof for a theorem of Thomas and Vasquez. J. Nb. Th. 55 (1995), 197-208. MR 96m:11099

[Shi] T. Shintani. On evaluation of zeta functions of totally real algebraic number fields at non-positive integers. J. Fac. Sci. Univ. Tokyo 23 (1976), 393-417. MR 55:266

[Sie] C. L. Siegel. Lectures on Advanced Analytic Number Theory. Tata Institute of Fundamental Research, Bombay, 1965. MR 41:6760

[Wa] L.C. Washington. Introduction to Cyclotomic Fields. Springer-Verlag, Grad.Texts Math. 83, 1982; 2nd ed., 1997. MR 85g:11001; MR 97h:11130

[Zag] D. Zagier. A Kronecker limit formula for real quadratic fields. Math. Ann. 213 (1975),153184. MR 51:3123

Université de Caen, Campus 2, Département de Mathématiques, 14032 Caen CEDEX, France

E-mail address: louboutimath.unicaen.fr 